CRYSTALLOGRAPHIC COMMUNICATIONS
Received 15 November 2017

Accepted 15 November 2017

Edited by M. Zeller, Purdue University, USA

Keywords: crystal structure; europium; pyrazolylborate; scorpionate; pyrazabole.

CCDC references: $1585878 ; 1585877$

Supporting information: this article has supporting information at journals.iucr.org/e

\title{
Formation and structural characterization of a europium(II) mono(scorpionate) complex and a sterically crowded pyrazabole
}

\author{
Phil Liebing, ${ }^{a}$ Marcel Kühling, ${ }^{\mathrm{b}}$ Josef Takats, ${ }^{\mathrm{c}}$ Liane Hilfert ${ }^{\mathrm{b}}$ and Frank T. \\ Edelmann $^{\mathbf{b}} *$
}

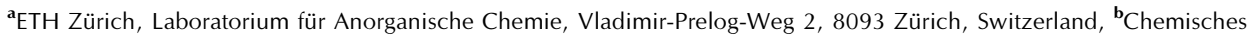
Institut der Otto-von-Guericke-Universität Magdeburg, Universitätsplatz 2, 39106 Magdeburg, Germany, and ${ }^{\mathbf{c}}$ Department of Chemistry, University of Alberta, Edmonton, Alberta, AB, Canada, T6G 2G2. *Correspondence e-mail: frank.edelmann@ovgu.de

The reaction of $\mathrm{EuI}_{2}(\mathrm{THF})_{2}$ with potassium hydrotris(3,5-diisopropylpyrazolyl)borate $\left(\mathrm{K}\left[\mathrm{HB}\left(3,5-{ }^{i \operatorname{Pr} 2} \mathrm{pz}\right)_{3}\right]\left(=\mathrm{KT} \mathrm{p}^{i \operatorname{Pr} 2}, \mathrm{pz}=\right.\right.$ pyrazolyl $)$ in a molar ratio of $1: 1.5$ resulted in extensive ligand fragmentation and formation of the europium(II) mono(scorpionate) complex bis(3,5-diisopropyl-1 $H$-pyrazole)[hydrotris(3,5diisopropylpyrazolyl)borato]iodidoeuropium(II), [ $\mathrm{Eu}\left(\mathrm{C}_{27} \mathrm{H}_{46} \mathrm{BN}_{6}\right) \mathrm{I}\left(\mathrm{C}_{9} \mathrm{H}_{16} \mathrm{~N}_{2}\right)_{2}$ ] or $\left(\mathrm{Tp}^{i \operatorname{Pr} 2}\right)\left(3,5-{ }^{i \operatorname{Pr} 2} \mathrm{pzH}\right)_{2} \mathrm{Eu}^{\mathrm{II}} \mathrm{I}, \mathbf{1}$, in high yield $(78 \%)$. As a typical by-product, small amounts of the sterically crowded pyrazabole derivative trans-4,8-bis(3,5diisopropylpyrazol-1-yl)-1,3,5,7-tetraisopropylpyrazabole, $\mathrm{C}_{36} \mathrm{H}_{62} \mathrm{~B}_{2} \mathrm{H}_{8}$ or trans$\left\{\left(3,5-{ }^{i \operatorname{Pr} 2} \mathrm{pz}\right) \mathrm{HB}\left(\mu-3,5{ }^{-}{ }^{\operatorname{Pr} 2} \mathrm{pz}\right)\right\}_{2}, 2$, were formed. Both title compounds have been structurally characterized through single-crystal X-ray diffraction. In $\mathbf{1}$, two isopropyl groups are each disordered over two orientations with occupancy ratios of $0.574(10): 0.426(10)$ and $0.719(16): 0.281$ (16). In 2, one isopropyl group is similarly disordered, occupancy ratio 0.649 (9):0.351 (9).

\section{Chemical context}

The organometallic chemistry of divalent lanthanides provides fascinating structures such as the sandwich complexes $\operatorname{Ln}\left(\mathrm{C}_{5} \mathrm{Me}_{5}\right)_{2}\left(\operatorname{Ln}=\mathrm{Sm}, \mathrm{Eu}, \mathrm{Yb} ; \mathrm{C}_{5} \mathrm{Me}_{5}=\eta^{5}\right.$-pentamethylcyclopentadienyl). An unusual structural feature of the unsolvated lanthanide sandwich complexes $\operatorname{Ln}\left(\mathrm{C}_{5} \mathrm{Me}_{5}\right)_{2}$ (Fig. 1a, $L n=\mathrm{Sm}, \mathrm{Eu}, \mathrm{Yb}$ ) is their bent metallocene structure

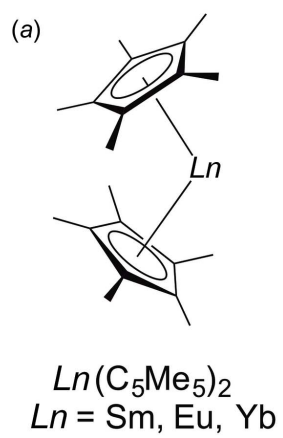

Figure 1

Comparison of the molecular structures of 'bent sandwich'-like lanthanide(II) cyclopentadienides ( $a$ ) and tris(3,5-diisopropylpyrazolyl)borates $(b)$. 
in the solid state. This opens up the coordination sphere around the central divalent lanthanide ions and accounts for the very high reactivity of these compounds (Evans et al., 1983, 1988; Evans, 2007). It has been demonstrated in the past that Trofimenko's famous hydrotris(pyrazolyl)borate ligands ('scorpionates') represent useful alternatives to the ubiquitous cyclopentadienyl ligands (Pettinari, 2008; Trofimenko, 1966, 1993, 1999). Like the cyclopentadienyl ligands, these tridentate, monoanionic ligands can also be largely varied in their steric demand by introducing different substituents in the 3- and 5-positions of the pyrazolyl rings. According to Trofimenko's nomenclature, the abbreviation $\mathrm{Tp}$ stands for the ring-unsubstituted hydrotris(pyrazolyl)borate, whereas e.g. $\mathrm{Tp}^{\mathrm{Me} 2}$ denotes the sterically more demanding hydrotris(3,5dimethylpyrazolyl)borate. The homoleptic divalent lanthanide complexes $\operatorname{Ln}\left(\mathrm{Tp}^{\mathrm{Me} 2}\right)_{2}(\operatorname{Ln}=\mathrm{Sm}, \mathrm{Eu}, \mathrm{Yb})$ have been found to adopt a highly symmetrical, trigonal-antiprismatic coordination comprising an almost linear $\mathrm{B} \cdots L n \cdots \mathrm{B}$ arrangement (Marques et al., 2002). Apparently, the sandwich-like structure of $\operatorname{Ln}\left(\mathrm{Tp}^{\mathrm{Me} 2}\right)_{2}$ is the result of the much larger cone angle of $\mathrm{Tp}^{\mathrm{Me} 2}\left(239^{\circ}\right)$ as compared to that of the $\mathrm{C}_{5} \mathrm{Me}_{5}$ ligand $\left(142^{\circ}\right)$ (Davies et al., 1985). More recently, these investigations have been successfully extended to the even larger hydrotris(3,5diisopropylpyrazolyl)borate ligand $\left(\mathrm{Tp}^{i \mathrm{Pr} 2}\right)$ (Kitajima et al., 1992). Homoleptic complexes of this ligand could be isolated with the 'classical' divalent lanthanides samarium, europium, thulium and ytterbium (Momin et al., 2014; Kühling et al., 2015). Rather surprisingly, crystal structure determinations revealed a 'bent sandwich'-like molecular structure like $\operatorname{Ln}\left(\mathrm{C}_{5} \mathrm{Me}_{5}\right)_{2}$ (Fig. 1b). Computational studies indicated that steric repulsion between the isopropyl groups forces the $\mathrm{Tp}{ }^{i \mathrm{Pr} 2}$ ligands apart and permits the formation of unusual interligand $\mathrm{C}-\mathrm{H} \cdots \mathrm{N}$ hydrogen-bonding interactions that help to stabilize the structure (Momin et al., 2014). The recently reported neon-yellow divalent europium complex $\mathrm{Eu}\left(\mathrm{Tp}^{i \mathrm{Pr} 2}\right)_{2}$ also stands out due to its bright-yellow photoluminescence, which has been investigated in great detail (Kühling et al., 2015; Suta et al., 2017). $\mathrm{Eu}\left(\mathrm{Tp}^{i \mathrm{Pr} 2}\right)_{2}$ was easily prepared in $83 \%$ yield by treatment of the bis-THF adduct of europium(II) diiodide, $\mathrm{EuI}_{2}(\mathrm{THF})_{2}$, with 2 equiv. of $\mathrm{KTp}^{i \mathrm{Pr} 2}$ in THF solution (Kühling et al. 2015). We now report that the use of a significantly smaller amount of $\mathrm{KTp}^{i \mathrm{Pr} 2}$ led to extensive ligand fragmentation and formation of the first europium(II) mono(scorpionate) complex, $\left[\mathrm{HB}\left(3,5{ }^{-}{ }^{P r 2} \mathrm{pz}\right)\right]\left(3,5^{-}{ }^{\mathrm{P} r} 2 \mathrm{pzH}\right){ }_{2} \mathrm{Eu}^{\mathrm{II} I}$ (1), in addition to a frequently observed by-product, the sterically crowded 4,8-bis(pyrazolyl)pyrazabole derivative trans$\left\{\left(3,5-{ }^{i \mathrm{Pr} 2} \mathrm{pz}\right) \mathrm{HB}\left(\mu-3,5{ }^{i \mathrm{Pr} 2} \mathrm{pz}\right)\right\}_{2}$ (2). Both products have been structurally characterized through single-crystal X-ray diffraction.

The starting material $\mathrm{EuI}_{2}(\mathrm{THF})_{2}$ was prepared from $\mathrm{Eu}$ metal and 1,2-diiodoethane using an established literature procedure (Girard et al., 1980). The reaction of $\mathrm{EuI}_{2}(\mathrm{THF})_{2}$ with 1.5 equiv. of $\mathrm{KTp}^{i \mathrm{Pr} 2}$ in THF produced a fluorescent, neon-yellow solution and a white precipitate of potassium iodide. Crystallization from $n$-pentane solvent afforded brightyellow, air-sensitive crystals, which turned out to be the unexpected europium(II) mono(scorpionate) complex
$\left(\mathrm{Tp}^{i \mathrm{Pr} 2}\right)\left(3,5{ }^{i \mathrm{Pr} 2} \mathrm{pzH}\right)_{2} \mathrm{Eu}^{\mathrm{II}} \mathrm{I}(\mathbf{1})$. The $78 \%$ isolated yield of $\mathbf{1}$ was surprisingly high. The coordinated neutral 3,5-diisopropylyrazole ligands clearly resulted from fragmentation of the $\mathrm{Tp}^{i \mathrm{Pr} 2}$ ligand. $L n$-induced fragmentation of substituted $\mathrm{Tp}$ ligands is well documented (Domingos et al., 2002, and references cited therein), but it seems to be even more prevalent in the sterically highly demanding $\mathrm{Tp}^{i \mathrm{Pr} 2}$ system, as seen in some recently reported $\operatorname{Ln}\left(\mathrm{Tp}^{i \mathrm{Pr} 2}\right)$-derived polysulfide complexes (Kühling et al., 2016). Despite its paramagnetic nature, interpretable NMR spectra could be obtained for 1. A single resonance at $\delta-5.3 \mathrm{ppm}$ in the ${ }^{11} \mathrm{~B}$ NMR spectrum proved the presence of a single boron-containing species. A high-intensity peak at $m / z 769$ in the mass spectrum of $\mathbf{1}$ could be assigned to the fragment ion $\left.\left[\mathrm{Eu}\left(\mathrm{Tp}^{i \mathrm{Pr} 2}\right){ }^{i \mathrm{Pr} 2} \mathrm{pz}\right)\right]^{+}$, while a peak at $m / z, 616$ corresponds to the ion $\left[\mathrm{Eu}\left(\mathrm{Tp}^{i \mathrm{Pr} 2}\right)\right]^{+}$.
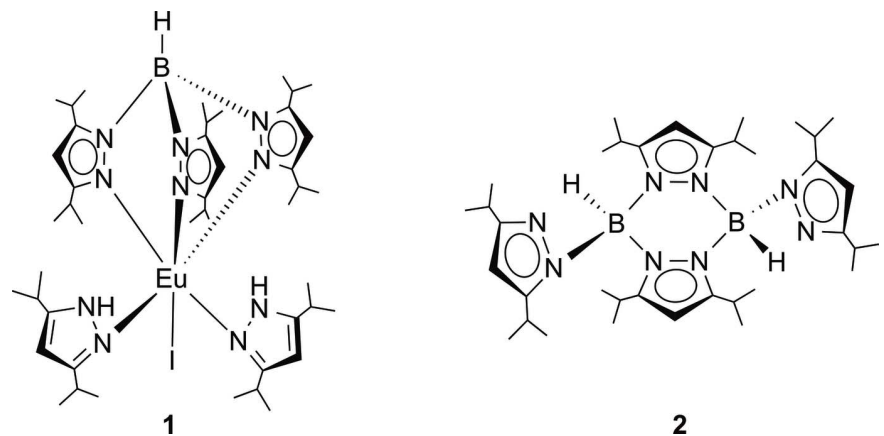

Further work-up of the supernatant solution remaining after isolation of $\mathbf{1}$ by addition of a large volume of non-polar hexamethyldisiloxane (HMDSO) resulted in the formation of well-formed, colorless, cube-like crystals in low yield. These turned out to be another ligand fragmentation product typical for lanthanide $\mathrm{Tp}$ chemistry, namely the 4,8-bis(pyrazolyl)pyrazabole derivative trans- $\left\{\left(3,5^{-}{ }^{i \mathrm{Pr} 2} \mathrm{pz}\right) \mathrm{HB}\left(\mu-3,5^{i \mathrm{Pr} 2} \mathrm{pz}\right)\right\}_{2}$ (2). The parent pyrazabole, $\left\{\mathrm{H}_{2} \mathrm{~B}(\mu-\mathrm{pz})\right\}_{2}$ has been known since 1966 when it was reported by Trofimenko contemporaneously with the discovery of Tp ligands (Trofimenko, 1966). Since then, numerous substituted pyrazaboles have been prepared and structurally investigated (Alcock \& Sawyer, 1974; Cavero et al., 2008; Niedenzu \& Niedenzu, 1984; Niedenzu \& Nöth, 1983; Trofimenko, 1966). In a number of recent studies, it has been demonstrated that certain substituted pyrazaboles possess unique photophysical and electrochemical properties and could thus find promising applications in organic photovoltaics (OPVs) and non-linear optics (Jadhav et al., 2013, 2015; Misra et al., 2013, 2014; Patil et al., 2017). Compound 2 belongs to the rather special class of 4,8-bis(pyrazolyl)pyrazaboles in which two hydrogen atoms at boron are replaced by pyrazolyl moieties (Niedenzu \& Niedenzu, 1984). Deliberate formation of the parent 4,8-bis(pyrazolyl)pyrazabole, 4,8-trans $-\{(\mathrm{pz}) \mathrm{HB}(\mu-\mathrm{pz})\}_{2}$, has been achieved by thermolysis of the free acid of the hydrotris(pyrazolyl)borate anion (Kresínski, 1999). In lanthanide Tp chemistry, such 4,8(pyrazolyl)pyrazaboles normally represent unwanted sideproducts as they frequently result from ligand fragmentation and are often the first crystalline products to come out of reaction mixtures (Kühling et al., 2015, 2016; Lobbia et al., 


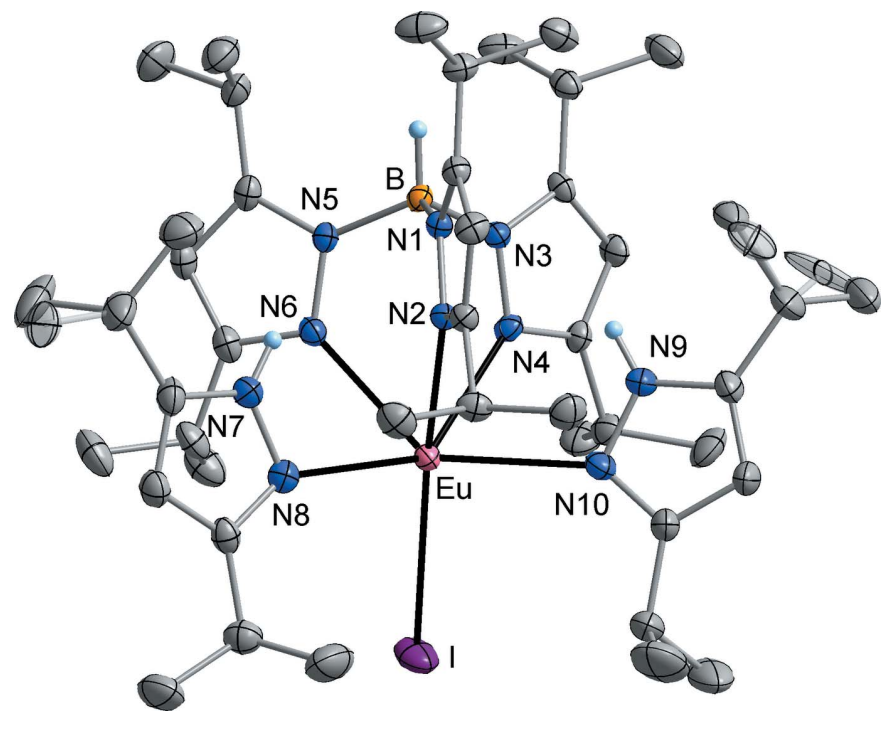

Figure 2

The molecular structure of compound $\mathbf{1}$ in the crystal, showing orientational disorder of two isopropyl groups. Displacement ellipsoids are drawn at the $40 \%$ probability level, $\mathrm{H}$ atoms attached to $\mathrm{C}$ atoms omitted for clarity.

1992). Spectroscopic characterization of $\mathbf{2}$ was in good agreement with the results of the X-ray diffraction study. For instance, the mass spectrum of $\mathbf{2}$ showed the molecular ion at $m / z 627$, and the ${ }^{11} \mathrm{~B}$ NMR spectrum displayed a single resonance at $\delta-4.3 \mathrm{ppm}$.

\section{Structural commentary}

Both title compounds $\mathbf{1}$ and $\mathbf{2}$ exist as well-separated molecules in the crystal. In the $\mathrm{Eu}^{\mathrm{II}}$ complex $\mathbf{1}$, one molecule is present in the asymmetric unit (Fig. 2). The $\mathrm{Tp}^{i \operatorname{Pr} 2}$ ligand is attached to $\mathrm{Eu}$ in a symmetric tridentate mode with an $\mathrm{H}-$ $\mathrm{B} \cdots$ Eu angle of $179.0(2)^{\circ}$. The three $\mathrm{Eu}-\mathrm{N}$ bonds cover the range 2.581 (2)-2.633 (2) $\AA$, which resembles that observed in the corresponding homoleptic $\mathrm{Eu}^{\mathrm{II}}$ complex $\mathrm{Eu}\left(\mathrm{Tp}^{i \mathrm{Pr} 2}\right)_{2}$ [2.563 (5)-2.670 (5) ̊; Suta et al., 2017]. The same applies to the $\mathrm{B}-\mathrm{N}$ bonds, which are in the narrow range 1.547 (4)1.555 (4) $\AA\left[\mathrm{Eu}\left(\mathrm{Tp}^{i \mathrm{Pr} 2}\right)_{2}: \mathrm{B}-\mathrm{N}=1.531\right.$ (8)-1.559 (7) $\left.\mathrm{A}\right]$. In $\mathbf{1}$, the coordination of the iodido ligand relative to the $\left(\mathrm{Tp}^{i \operatorname{Pr} 2}\right)^{-}$ ligand is slightly tilted $\left[\mathrm{I}-\mathrm{Eu} \cdots \mathrm{B}=151.49(5)^{\circ}\right]$, and an almost linear arrangement of the iodido ligand and one of the $T \mathrm{p}^{i \operatorname{Pr} 2} \mathrm{~N}$-donor atoms is realized $\left[\mathrm{I}-\mathrm{Eu}-\mathrm{N} 2=165.92(5)^{\circ}\right] . \mathrm{A}$ strongly distorted octahedral coordination is completed by the two neutral $\left(3,5^{i \mathrm{Pr} 2} \mathrm{pzH}\right)$ ligands, with coordination angles of $138.80(7)^{\circ}(\mathrm{N} 4-\mathrm{Eu}-\mathrm{N} 8)$ and $137.43(7)^{\circ}(\mathrm{N} 6-\mathrm{Eu}-\mathrm{N} 10)$. The corresponding $\mathrm{Eu}-\mathrm{N}$ bond lengths $[\mathrm{Eu}-\mathrm{N} 8=2.699$ (3), $\mathrm{Eu}-\mathrm{N} 10=2.660$ (2) $\AA$ ] are slightly longer than those to the $\left(\mathrm{T} \mathrm{p}^{i \mathrm{Pr} 2}\right)^{-}$ligand, which may be due to the absence of negative ligand charge. The $\mathrm{NH} \cdots \mathrm{N}$ distances between the two pyrazole NH moieties and potential hydrogen-acceptor atoms (N2, N4, N6) are in the range 2.512 (2)-2.610 (2) $\AA$, but the groups are not in a proper orientation for efficient hydrogen bonding $\left[\mathrm{N}-\mathrm{H} \cdots \mathrm{N} 115.0(2)-122.0(2)^{\circ}\right]$. Consequently, stabilization of the molecular structure by intramolecular hydrogen bonding is presumably of less importance.

The pyrazabol $\mathbf{2}$ exists as a centrosymmetric dimer in the crystal, which formally results from two $\mathrm{HB}\left(3,5^{-}{ }^{i \operatorname{Pr} 2} \mathrm{pz}\right)_{2}$ monomers (Fig. 3). The two B atoms are interconnected by two $\mu$-bridging $\left(3,5-{ }^{i \mathrm{Pr} 2} \mathrm{pz}\right)$ moieties, resulting in a planar, sixmembered $\mathrm{B}_{2} \mathrm{~N}_{4}$ ring. The $\mathrm{B}-\mathrm{N}$ bonds within this ring are virtually equal at 1.554 (2) $\AA$ (B-N1) and 1.557 (2) $\AA$ (B$\left.\mathrm{N} 2^{\prime}\right)$, and therefore similar to that within the $\left(\mathrm{Tp}^{i \operatorname{Pr} 2}\right)^{-}$ligand in 1. In contrast, the $\mathrm{B}-\mathrm{N}$ bond to the terminal $\left(3,5^{i \mathrm{Pr} 2} \mathrm{pz}\right)$ moiety $(\mathrm{B}-\mathrm{N} 3)$ is slightly shortened to 1.532 (2) $\AA$. The B atoms in $\mathbf{2}$ exhibit a virtually ideal tetrahedral coordination with bonding angles in the narrow range $108.3(1)-110.7(1)^{\circ}$. The molecular structure of $\mathbf{2}$ is very similar to that of the 3,5-dimethylpyrazolyl analog, trans $-\left\{\left(3,5-{ }^{\mathrm{Me} 2} \mathrm{pz}\right) \mathrm{HB}(\mu-\right.$ $\left.\left.3,5-{ }^{\mathrm{Me} 2} \mathrm{pz}\right)\right\}_{2} \quad[\mathrm{~B}-\mathrm{N}=1.5419(2)$ and $1.5486(1) \AA$ for $\mu-$ $\left(3,5-{ }^{\mathrm{Me} 2} \mathrm{pz}\right)$ and 1.5257 (2) $\AA$ for terminal $3,5-{ }^{\mathrm{Me} 2} \mathrm{pz}, \mathrm{N}-\mathrm{B}-\mathrm{N}$ $=108.532$ (6)-109.091 (6) ${ }^{\circ}$; Alcock \& Sawyer, 1974]. In contrast, the unsubstituted pyrazabol trans $-\{(\mathrm{pz}) \mathrm{HB}(\mu-\mathrm{pz})\}_{2}$ is non-centrosymmetric and features a remarkably puckered $\mathrm{B}_{2} \mathrm{~N}_{4}$ ring $[\mathrm{B}-\mathrm{N}=1.546(3)-1.559(3) \AA$ for $\mu-\mathrm{pz}$ and 1.501 (3)-1.533 (3) $\AA$ for terminal pz, N-B-N = 105.2 (2)111.0 (2) ${ }^{\circ}$; Kresiński, 1999].

\section{Supramolecular features}

In both compounds $\mathbf{1}$ and $\mathbf{2}$, no unusually short intermolecular contacts have been observed. In 1, the bulky ${ }^{i} \operatorname{Pr}$ groups at the molecule's surface does not allow for intermolecular $\mathrm{N}-$ $\mathrm{H} \cdots \mathrm{N}$ hydrogen bonding.

\section{Database survey}

For selected references on the reactivity of the sandwich complexes $\operatorname{Ln}\left(\mathrm{C}_{5} \mathrm{Me}_{5}\right)_{2}(\operatorname{Ln}=\mathrm{Sm}, \mathrm{Eu}, \mathrm{Yb})$, see: Evans et al. (1983, 1988), Evans (2007).

For general information on scorpionate ligands, see: Kitajima et al. (1992), Pettinari (2008), Trofimenko (1966, 1999).

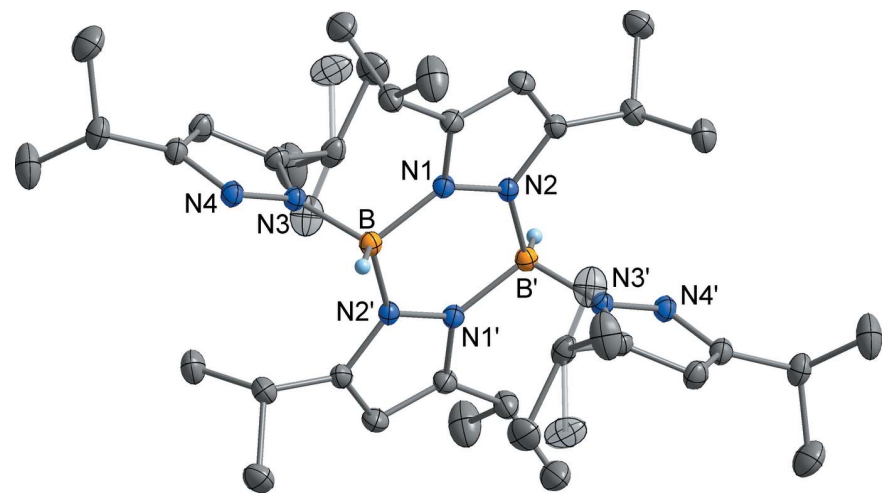

Figure 3

The molecular structure of compound $\mathbf{2}$ in the crystal, showing orientational disorder of one isopropyl group. Displacement ellipsoids drawn at the $50 \%$ probability level, $\mathrm{H}$ atoms attached to $\mathrm{C}$ atoms omitted for clarity. [Symmetry code: (') $\frac{1}{2}-x, \frac{1}{2}-y,-z$.] 
Table 1

Experimental details.

1

Crystal data

Chemical formula

$M_{\mathrm{r}}$

Crystal system, space group

Temperature $(\mathrm{K})$

$a, b, c(\AA)$

$\alpha, \beta, \gamma\left(^{\circ}\right)$

$V\left(\AA^{3}\right)$

Z

Radiation type

$\mu\left(\mathrm{mm}^{-1}\right)$

Crystal size (mm)

Data collection

Diffractometer

Absorption correction

$T_{\min }, T_{\max }$

No. of measured, independent and

observed $[I>2 \sigma(I)]$ reflections

$R_{\text {int }}$

$(\sin \theta / \lambda)_{\max }\left(\AA^{-1}\right)$

Refinement

$R\left[F^{2}>2 \sigma\left(F^{2}\right)\right], w R\left(F^{2}\right), S$

No. of reflections

No. of parameters

No. of restraints

$\mathrm{H}$-atom treatment

$\Delta \rho_{\max }, \Delta \rho_{\min }\left(\mathrm{e} \AA^{-3}\right)$

\author{
$\left[\mathrm{Eu}\left(\mathrm{C}_{27} \mathrm{H}_{46} \mathrm{BN}_{6}\right) \mathrm{I}\left(\mathrm{C}_{9} \mathrm{H}_{16} \mathrm{~N}_{2}\right)_{2}\right]$ \\ 1048.84 \\ Orthorhombic, $\mathrm{Pbca}$ \\ 153 \\ 19.5319 (4), 26.6614 (4), 19.8681 (3) \\ 90, 90,90 \\ $10346.3(3)$ \\ 8 \\ Mo $K \alpha$ \\ 1.85 \\ $0.49 \times 0.27 \times 0.21$
}

Stoe IPDS $2 \mathrm{~T}$

Numerical ( $X$-AREA and $X$-RED; Stoe \& Cie, 2002)

$0.535,0.716$

43898, 10158, 8229

0.045

0.617

$0.031,0.063,1.04$

10158

576

24

$\mathrm{H}$-atom parameters constrained

$1.24,-1.27$
2

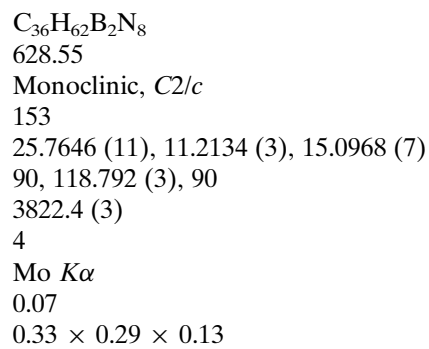

Stoe IPDS 2T

$10509,3367,2594$

0.046

0.595

$0.044,0.104,1.02$

3367

238

0

$\mathrm{H}$-atom parameters constrained $0.25,-0.21$

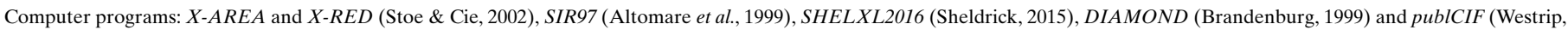
2010).

For the chemistry of divalent lanthanide scorpionate complexes, see: Davies et al. (1985), Domingos et al. (2002), Hillier et al. (2001), Kühling et al. (2015, 2016), Marques et al. (2002), Momin et al. (2014), Suta et al. (2017).

For general information on the chemistry and structures of pyrazaboles, see: Cavero et al. (2008), Niedenzu \& Niedenzu (1984), Niedenzu \& Nöth (1983), Trofimenko (1966).

For information on practical applications of pyrazaboles, see: Jadhav et al. (2013, 2015), Misra et al. (2013, 2014), Patil et al. (2017).

\section{Synthesis and crystallization}

All operations were performed under an argon atmosphere using standard Schlenk techniques. THF, hexamethyldisiloxane (HMDSO), and $n$-pentane were distilled from sodium/benzophenone under argon prior to use. NMR spectra were recorded on a Bruker DPX400 $\left({ }^{1} \mathrm{H}: \quad 400 \mathrm{MHz}\right)$ spectrometer in THF- $D_{8}$ at 295 (2) K. The ${ }^{11} \mathrm{~B}$ NMR spectra were obtained by using inverse gated decoupling on a Bruker Avance $400 \mathrm{NMR}$ spectrometer, operating at $128.4 \mathrm{MHz}$. The external standard was $15 \mathrm{wt}-\% \mathrm{BF}_{3} \cdot \mathrm{OEt}_{2}$ in $\mathrm{CDCl}_{3}\left(\delta_{\mathrm{B}}=0\right.$ ppm). IR spectra were measured on a Bruker Vertex V70 spectrometer equipped with a diamond ATR unit, electron impact mass spectra on a MAT95 spectrometer with an ionization energy of $70 \mathrm{eV}$. Elemental analyses $(\mathrm{C}, \mathrm{H}$ and $\mathrm{N})$ were performed using a VARIO EL cube apparatus. The starting materials $\mathrm{EuI}_{2}(\mathrm{THF})_{2}$ (Girard et al. 1980) and $\mathrm{KTp}^{i \operatorname{Pr} 2}$ (Kitajima et al. 1992) were prepared according to published procedures.

Preparation of $\left(\mathrm{Tp}^{i \operatorname{Pr} 2}\right)\left(3,5-{ }^{i \operatorname{Pr} 2} \mathrm{Hpz}\right)_{2} \mathrm{Eu}^{\mathrm{II} \mathrm{I}}$ (1) and trans$\left\{\left(3,5-{ }^{i \mathrm{Pr} 2} \mathrm{pz}\right) \mathrm{HB}\left(\mu-3,5^{-}{ }^{i \mathrm{Pr} 2} \mathrm{pz}\right)\right\}_{2}(\mathbf{2})$ : In a $250 \mathrm{~mL}$ Schlenk flask, THF $(150 \mathrm{~mL})$ was added to a mixture of $\mathrm{EuI}_{2}(\mathrm{THF})_{2}(2.36 \mathrm{~g}$, $4.29 \mathrm{mmol})$ and $\mathrm{KTp}^{i \operatorname{Pr} 2}(3.20 \mathrm{~g}, 6.33 \mathrm{mmol})$, and the resulting suspension was stirred for $12 \mathrm{~h}$ at r.t. A white precipitate (KI) was removed by filtration and the neon-yellow, fluorescent filtrate was evaporated to dryness. The residue was extracted with $n$-pentane $(3 \times 50 \mathrm{~mL})$, the combined extracts filtered again and concentrated in vacuo to a total volume of $c a 30 \mathrm{~mL}$. Cooling to $277 \mathrm{~K}$ afforded bright-yellow, air-sensitive crystals of $\mathbf{1}(3.64 \mathrm{~g}, 78 \%)$, which were suitable for X-ray diffraction. The mother liquid was taken to dryness, and the slightly sticky residue was redissolved in $c a 5 \mathrm{~mL}$ of THF. Addition of dry hexamethyldisiloxane ( $c$ a $50 \mathrm{~mL}$ ) followed by cooling to $277 \mathrm{~K}$ for several days afforded $c a 0.5 \mathrm{~g}$ of $\mathbf{2}$ as colorless, cube-like single-crystals.

1: Analysis calculated for $\mathrm{C}_{45} \mathrm{H}_{78} \mathrm{BEuIN}_{10}, \quad M=$ 1049.86 $\mathrm{g} \mathrm{mol}^{-1}$ : C 51.48, H 7.58, N 13.34\%. Found: C 50.88, H 7.77, N 12.59\%. M.p. ca 353 K (dec.). IR: v $3173 w, 3096 w$ (v C-H pyrazolyl), $2961 s, 2929 m, 2869 m\left(v \mathrm{CH}_{3}\right), 2550 w$ (vB-H), $1565 w, 1534 m, 1460 s, 1426 m, 1379 s, 1361 s, 1295$ $m, 1170 v s, 1104 m, 1046 s, 1012 s, 958 w, 923 w, 878 w, 787 v s$, $767 s, 716 m, 659 s, 587 w, 511 w, 462 w, 389 w, 362 w, 306 w$, $258 w, 219 w, 109 s, 75 \mathrm{~m} \mathrm{~cm}^{-1.1} \mathrm{H}$ NMR (400.1 MHz, THF- $D_{8}$, 
$300 \mathrm{~K}): \delta 11.6(s b r, \mathrm{~B}-H), 5.70(s b r, 5 \mathrm{H}, \mathrm{C}-H$ pyrazolyl $), 2.88$ $\delta 153.8$ (br, $q$ - $C$ pyrazolyl), 98.7, 99.3 ( $C-\mathrm{H}$ pyrazolyl), 27.9, $32.1\left(C-\mathrm{H}{ }^{i} \mathrm{Pr}\right), 23.2\left(\mathrm{CH}_{3}{ }^{i} \mathrm{Pr}\right) .{ }^{11} \mathrm{~B}$ NMR $\left(300 \mathrm{~K}\right.$, THF- $D_{8}$, $128.4 \mathrm{MHz}): \delta-5.3(s, b r)$ ppm. MS: $m / z$ (\%) $769(98)$ $\left[\mathrm{Eu}\left(\mathrm{Tp}^{i} \mathrm{Pr}_{2}\right)\left({ }^{i \mathrm{Pr} 2} \mathrm{pz}\right)\right]^{+}, \quad 616(92) \quad\left[\mathrm{Eu}\left(\mathrm{Tp}^{i \mathrm{Pr} 2}\right)\right]^{+}, \quad 477(85)$, $\left.321(100), 302(55)\left[\mathrm{EuBH}\left({ }^{i \mathrm{Pr} 2} \mathrm{pz}-\mathrm{CH}_{3}\right)\right]^{+}, 152(21){ }^{[\mathrm{Pr} 2} \mathrm{pz}\right]^{+}$, 137 (63).

2: Analysis calculated for $\mathrm{C}_{36} \mathrm{H}_{62} \mathrm{~B}_{2} \mathrm{~N}_{8}, M=628.56 \mathrm{~g} \mathrm{~mol}^{-1}$ : C 68.79, H 9.94, N 17.83\%. Found: C 68.50, H 10.10, N 17.53\%. M.p. 553 K. IR: $v 3176 w, 3094 w$ (v C-H pyrazolyl), $2966 s$, $2928 m, 2869 m, 2825 w\left(v \mathrm{CH}_{3}\right), 2467 w(v \mathrm{~B}-\mathrm{H}), 1576 w$, $1541 m, 1497 m, 1461 m, 1369 m, 1301 s, 1233 v s, 1169 v s, 1134$ $v s, 1090 s, 1063 s, 1041 \mathrm{~m}, 1015 \mathrm{~m}, 982 \mathrm{~s}, 919 w, 879 w, 832 s$, $788 s, 751 m, 723 m, 675 m, 566 m, 508 m, 473 w, 365 m, 302 m$, $246 m, 137 m, 106 m, 75 \mathrm{~m} \mathrm{~cm}^{-1 .}{ }^{1} \mathrm{H}$ NMR $(400.1 \mathrm{MHz}$, THF$\left.D_{8}, 300 \mathrm{~K}\right): \delta 11.0(s b r, 2 \mathrm{H}, \mathrm{B}-H), 5.75(s b r, 4 \mathrm{H}, \mathrm{C}-H$ pyrazolyl), 2.87-2.91 ( $\left.m, 4 \mathrm{H}, \mathrm{C}-H^{i} \mathrm{Pr}\right), 1.15-1.27(m, 48 \mathrm{H}$, $\mathrm{CH}_{3}{ }^{\mathrm{i}} \mathrm{Pr}$ ) ppm. ${ }^{13} \mathrm{C}$ NMR $\left(300 \mathrm{~K}\right.$, THF- $\left.D_{8}, 100 \mathrm{MHz}\right): \delta 160.7$ (br, q-C pyrazolyl), $97.5\left(C-\mathrm{H}\right.$ pyrazolyl), $28.5\left(C-\mathrm{H}^{i} \mathrm{Pr}\right)$, $23.5\left(\mathrm{CH}_{3}{ }^{i} \mathrm{Pr}\right) .{ }^{11} \mathrm{~B}$ NMR $\left(300 \mathrm{~K}\right.$, THF- $\left.D_{8}, 128.4 \mathrm{MHz}\right): \delta-4.3$ $(s, \quad b r) \quad$ ppm. MS: $m / z$ (\%) $627(62) \quad[M]^{+}, \quad 476(100)$ $\left[\mathrm{C}_{27} \mathrm{H}_{46} \mathrm{~B}_{2} \mathrm{~N}_{6}\right]^{+}, \quad 461(75) \quad\left[\mathrm{C}_{26} \mathrm{H}_{43} \mathrm{~B}_{2} \mathrm{~N}_{6}\right]^{+}, \quad 325$ (66) $\left[\mathrm{C}_{18} \mathrm{H}_{31} \mathrm{~B}_{2} \mathrm{~N}_{4}\right]^{+}, 152$ (74) $\left[\mathrm{C}_{6} \mathrm{H}_{2} \mathrm{~B}_{2} \mathrm{~N}_{4}\right]^{+}, 137$ (89) $\left[\mathrm{pz}_{2}\right]$.

\section{Refinement}

Crystal data, data collection and structure refinement details are summarized in Table 1. All $\mathrm{H}$ atoms were refined as riding atoms with $\mathrm{B}-\mathrm{H}=1.00 \AA$ and $\mathrm{C}-\mathrm{H}=0.98-1.00 \AA$ and $U_{\text {iso }}(\mathrm{H})=1.5 U_{\text {eq }}(\mathrm{C})$ for methyl $\mathrm{H}$ atoms and $U_{\text {iso }}(\mathrm{H})=$ $1.2 U_{\text {eq }}(\mathrm{B}, \mathrm{C})$ for all others. In $\mathbf{1}$, two isopropyl groups are each disordered over two orientations with occupancy ratios of 0.574 (10):0.426 (10) and 0.719(16):0.281 (16). In 2, one isopropyl group is similarly disordered, occupancy ratio 0.649 (9):0.351 (9).

\section{Funding information}

General financial support of this work by the Otto-vonGuericke-Universität is gratefully acknowledged.

\section{References}

Alcock, N. W. \& Sawyer, J. F. (1974). Acta Cryst. B30, 2899-2901. Altomare, A., Burla, M. C., Camalli, M., Cascarano, G. L., Giacovazzo, C., Guagliardi, A., Moliterni, A. G. G., Polidori, G. \& Spagna, R. (1999). J. Appl. Cryst. 32, 115-119.

Brandenburg, K. (1999). DIAMOND. Crystal Impact GbR, Bonn, Germany.

Cavero, E., Giménez, R., Uriel, S., Beltrán, E., Serrano, J. L., Alkorta, I. \& Elguero, J. (2008). Cryst. Growth Des. 8, 838-847.
Davies, C. E., Gardiner, I. M., Green, J. C., Green, M. L. H., Hazel, N. J., Grebenik, P. D., Mtetwa, V. S. B. \& Prout, H. (1985). J. Chem. Soc. Dalton Trans. pp. 669-683.

Domingos, Â., Elsegood, M. R. J., Hillier, A. C., Lin, G., Liu, S. Y., Lopes, I., Marques, N., Maunder, G. H., McDonald, R., Sella, A., Steed, J. W. \& Takats, J. (2002). Inorg. Chem. 41, 6761-6768.

Evans, W. J. (2007). Inorg. Chem. 46, 3435-3449.

Evans, W. J., Bloom, I., Hunter, W. E. \& Atwood, J. L. (1983). J. Am. Chem. Soc. 105, 1401-1403.

Evans, W. J., Ulibarri, T. A. \& Ziller, J. W. (1988). J. Am. Chem. Soc. 110, 6877-6879.

Girard, P., Namy, J. L. \& Kagan, H. B. (1980). J. Am. Chem. Soc. 102, 2693-2698.

Hillier, A. C., Zhang, X. W., Maunder, G. H., Liu, S. Y., Eberspacher, T. A., Metz, M. V., McDonald, R., Domingos, Â., Marques, N., Day, V. W., Sella, A. \& Takats, J. (2001). Inorg. Chem. 40, 51065116.

Jadhav, T., Dhokale, B., Patil, Y. \& Misra, R. (2015). RSC Adv. 5, 68187-68191.

Jadhav, T., Maragani, R., Misra, R., Sreeramulu, Y., Rao, D. N. \& Mobin, S. M. (2013). Dalton Trans. 42, 4340-4342.

Kitajima, N., Fujisawa, K., Fujimoto, C., Morooka, Y., Hashimoto, S., Kitagawa, T., Toriumi, K., Tatsumi, K. \& Nakamura, A. (1992). J. Am. Chem. Soc. 114, 1277-1291.

Kresiński, R. A. (1999). J. Chem. Soc. Dalton Trans. pp. 401-406.

Kühling, M., McDonald, R., Liebing, P., Hilfert, L., Ferguson, M. J., Takats, J. \& Edelmann, F. T. (2016). Dalton Trans. 45, 1011810121.

Kühling, M., Wickleder, C., Ferguson, M. J., Hrib, C. G., McDonald, R., Suta, M., Hilfert, L., Takats, J. \& Edelmann, F. T. (2015). New J. Chem. 39, 7617-7625.

Lobbia, G. G., Bonati, F., Cecchi, P. \& Pettinari, C. (1992). Gazz. Chim. Ital. 121, 355-358.

Marques, N., Sella, A. \& Takats, J. (2002). Chem. Rev. 102, 2137-2160.

Misra, R., Jadhav, T. \& Mobin, S. M. (2013). Dalton Trans. 42, 16614 16620.

Misra, R., Jadhav, T. \& Mobin, S. M. (2014). Dalton Trans. 43, $2013-$ 2022.

Momin, A., Carter, L., Yang, Y., McDonald, R., Essafi (née Labouille), S., Nief, F., Del Rosal, I., Sella, A., Maron, L. \& Takats, J. (2014). Inorg. Chem. 53, 12066-12075.

Niedenzu, K. \& Niedenzu, P. M. (1984). Inorg. Chem. 23, 37133716.

Niedenzu, K. \& Nöth, H. (1983). Chem. Ber. 116, 1132-1153.

Patil, Y., Jadhav, T., Dhokale, B., Butenschön, H. \& Misra, R. (2017). ChemistrySelect 2, 415-420.

Pettinari, C. (2008). Scorpionates II: Chelating Borate Ligands. Imperial College Press, London.

Sheldrick, G. M. (2015). Acta Cryst. C71, 3-8.

Stoe \& Cie (2002). $X$-AREA and $X$-RED. Stoe \& Cie, Darmstadt, Germany.

Suta, M., Kühling, M., Liebing, P., Edelmann, F. T. \& Wickleder, C. (2017). J. Lumin. 187, 62-68.

Trofimenko, S. (1966). J. Am. Chem. Soc. 88, 1842-1844.

Trofimenko, S. (1993). Chem. Rev. 93, 943-980.

Trofimenko, S. (1999). The Coordination Chemistry of Scorpionates Pyrazolylborate Ligands. Imperial College Press, London.

Westrip, S. P. (2010). J. Appl. Cryst. 43, 920-925. 


\section{supporting information}

Acta Cryst. (2017). E73, 1921-1925 [https://doi.org/10.1107/S2056989017016498]

\section{Formation and structural characterization of a europium(II) mono(scorpionate) complex and a sterically crowded pyrazabole}

Phil Liebing, Marcel Kühling, Josef Takats, Liane Hilfert and Frank T. Edelmann

Computing details

For both structures, data collection: $X$-AREA (Stoe \& Cie, 2002); cell refinement: $X$ - $A R E A$ (Stoe \& Cie, 2002); data reduction: $X$-AREA and $X$-RED (Stoe \& Cie, 2002); program(s) used to solve structure: SIR97 (Altomare et al., 1999); program(s) used to refine structure: SHELXL2016 (Sheldrick, 2015); molecular graphics: DIAMOND (Brandenburg, 1999); software used to prepare material for publication: publCIF (Westrip, 2010).

Bis(3,5-diisopropyl-1 H-pyrazole) [hydrotris(3,5-diisopropylpyrazolyl)borato]iodidoeuropium(II) (1)

Crystal data

$\left[\mathrm{Eu}\left(\mathrm{C}_{27} \mathrm{H}_{46} \mathrm{BN}_{6}\right) \mathrm{I}\left(\mathrm{C}_{9} \mathrm{H}_{16} \mathrm{~N}_{2}\right)_{2}\right]$

$M_{r}=1048.84$

Orthorhombic, $\mathrm{Pbca}$

$a=19.5319$ (4) $\AA$

$b=26.6614$ (4) $\AA$

$c=19.8681(3) \AA$

$V=10346.3(3) \AA^{3}$

$Z=8$

$F(000)=4312$

Data collection

Stoe IPDS 2T

diffractometer

Radiation source: fine-focus sealed tube

Detector resolution: 6.67 pixels $\mathrm{mm}^{-1}$

area detector scans

Absorption correction: numerical

(X-AREA and X-RED; Stoe \& Cie, 2002)

$T_{\min }=0.535, T_{\max }=0.716$

Refinement

Refinement on $F^{2}$

Least-squares matrix: full

$R\left[F^{2}>2 \sigma\left(F^{2}\right)\right]=0.031$

$w R\left(F^{2}\right)=0.063$

$S=1.04$

10158 reflections

576 parameters

24 restraints

Primary atom site location: heavy-atom method
$D_{\mathrm{x}}=1.347 \mathrm{Mg} \mathrm{m}^{-3}$

Mo $K \alpha$ radiation, $\lambda=0.71073 \AA$

Cell parameters from 44511 reflections

$\theta=2.2-26.2^{\circ}$

$\mu=1.85 \mathrm{~mm}^{-1}$

$T=153 \mathrm{~K}$

Block, yellow

$0.49 \times 0.27 \times 0.21 \mathrm{~mm}$

43898 measured reflections

10158 independent reflections

8229 reflections with $I>2 \sigma(I)$

$R_{\text {int }}=0.045$

$\theta_{\max }=26.0^{\circ}, \theta_{\min }=2.2^{\circ}$

$h=-23 \rightarrow 24$

$k=-32 \rightarrow 32$

$l=-21 \rightarrow 24$

Secondary atom site location: difference Fourier map

Hydrogen site location: inferred from neighbouring sites

$\mathrm{H}$-atom parameters constrained

$w=1 /\left[\sigma^{2}\left(F_{\mathrm{o}}^{2}\right)+(0.0226 P)^{2}+10.682 P\right]$

where $P=\left(F_{\mathrm{o}}{ }^{2}+2 F_{\mathrm{c}}{ }^{2}\right) / 3$

$(\Delta / \sigma)_{\max }=0.002$

$\Delta \rho_{\max }=1.24 \mathrm{e} \AA^{-3}$

$\Delta \rho_{\min }=-1.27$ e $\AA^{-3}$ 
Extinction correction: SHELXL2016

(Sheldrick, 2015),

$\mathrm{Fc}^{*}=\mathrm{kFc}\left[1+0.001 \times \mathrm{Fc}^{2} \lambda^{3} / \sin (2 \theta)\right]^{-1 / 4}$

Extinction coefficient: $0.00019(2)$

\section{Special details}

Geometry. All esds (except the esd in the dihedral angle between two 1.s. planes) are estimated using the full covariance matrix. The cell esds are taken into account individually in the estimation of esds in distances, angles and torsion angles; correlations between esds in cell parameters are only used when they are defined by crystal symmetry. An approximate (isotropic) treatment of cell esds is used for estimating esds involving l.s. planes.

Fractional atomic coordinates and isotropic or equivalent isotropic displacement parameters $\left(\AA^{2}\right)$

\begin{tabular}{|c|c|c|c|c|c|}
\hline & $x$ & $y$ & $z$ & $U_{\text {iso }} * / U_{\text {eq }}$ & Occ. $(<1)$ \\
\hline $\mathrm{C} 1$ & $0.23921(15)$ & $0.19065(10)$ & $0.45330(14)$ & $0.0232(6)$ & \\
\hline $\mathrm{C} 2$ & $0.21068(16)$ & 0.19034 (11) & $0.38902(14)$ & $0.0273(6)$ & \\
\hline $\mathrm{H} 4$ & 0.183276 & 0.215746 & 0.369057 & $0.033 *$ & \\
\hline $\mathrm{C} 3$ & $0.23046(15)$ & $0.14552(10)$ & $0.36038(14)$ & $0.0238(6)$ & \\
\hline $\mathrm{C} 4$ & $0.23355(17)$ & $0.23068(11)$ & $0.50638(15)$ & $0.0283(6)$ & \\
\hline H5 & 0.263908 & 0.221037 & 0.544789 & $0.034 *$ & \\
\hline $\mathrm{C} 5$ & 0.25808 (19) & $0.28129(12)$ & $0.47934(18)$ & $0.0384(8)$ & \\
\hline H8 & 0.255417 & 0.306493 & 0.515143 & $0.058^{*}$ & \\
\hline H6 & 0.228981 & 0.291544 & 0.441589 & $0.058^{*}$ & \\
\hline $\mathrm{H} 7$ & 0.305575 & 0.278252 & 0.463953 & $0.058^{*}$ & \\
\hline C6 & $0.16048(18)$ & $0.23508(12)$ & $0.53306(17)$ & $0.0381(8)$ & \\
\hline H10 & 0.158795 & 0.260725 & 0.568352 & $0.057 *$ & \\
\hline H9 & 0.146011 & 0.202746 & 0.551724 & $0.057^{*}$ & \\
\hline H11 & 0.129735 & 0.244581 & 0.496224 & $0.057^{*}$ & \\
\hline $\mathrm{C} 7$ & $0.21582(15)$ & $0.12724(11)$ & $0.29037(14)$ & $0.0291(6)$ & \\
\hline H12 & 0.218647 & 0.089789 & 0.290276 & $0.035^{*}$ & \\
\hline C8 & $0.14350(19)$ & $0.14246(15)$ & $0.26872(18)$ & $0.0492(9)$ & \\
\hline H14 & 0.133731 & 0.128534 & 0.224096 & $0.074 *$ & \\
\hline H13 & 0.140294 & 0.179121 & 0.266970 & $0.074 *$ & \\
\hline H15 & 0.110206 & 0.129474 & 0.301242 & $0.074^{*}$ & \\
\hline C9 & $0.2686(2)$ & 0.14745 (12) & $0.24108(16)$ & $0.0392(8)$ & \\
\hline H17 & 0.259015 & 0.134409 & 0.195949 & $0.059 *$ & \\
\hline H18 & 0.314471 & 0.136803 & 0.255155 & $0.059^{*}$ & \\
\hline H16 & 0.266440 & 0.184172 & 0.240395 & $0.059 *$ & \\
\hline $\mathrm{C} 10$ & 0.48118 (14) & 0.09139 (11) & $0.45682(14)$ & $0.0244(6)$ & \\
\hline C11 & $0.49553(15)$ & $0.07902(11)$ & $0.39050(14)$ & $0.0274(6)$ & \\
\hline H19 & 0.539399 & 0.077925 & 0.369911 & $0.033 *$ & \\
\hline $\mathrm{C} 12$ & $0.43383(15)$ & $0.06867(10)$ & $0.36050(14)$ & $0.0238(6)$ & \\
\hline $\mathrm{C} 13$ & $0.52990(15)$ & $0.10295(12)$ & $0.51323(15)$ & $0.0309(7)$ & \\
\hline $\mathrm{H} 20$ & 0.502309 & 0.114062 & 0.552928 & $0.037 *$ & \\
\hline C14 & $0.56970(19)$ & $0.05623(13)$ & $0.53359(17)$ & $0.0432(8)$ & \\
\hline $\mathrm{H} 21$ & 0.600301 & 0.064330 & 0.571137 & $0.065^{*}$ & \\
\hline $\mathrm{H} 22$ & 0.596805 & 0.044372 & 0.495250 & $0.065^{*}$ & \\
\hline $\mathrm{H} 23$ & 0.537614 & 0.029938 & 0.547469 & $0.065^{*}$ & \\
\hline C15 & $0.57849(19)$ & $0.14513(14)$ & $0.4948(2)$ & $0.0483(9)$ & \\
\hline
\end{tabular}




\begin{tabular}{|c|c|c|c|c|}
\hline $\mathrm{H} 24$ & 0.607370 & 0.153213 & 0.533668 & $0.073^{*}$ \\
\hline $\mathrm{H} 25$ & 0.552076 & 0.174828 & 0.481785 & $0.073^{*}$ \\
\hline $\mathrm{H} 26$ & 0.607449 & 0.134594 & 0.457093 & $0.073 *$ \\
\hline $\mathrm{C} 16$ & $0.42087(16)$ & $0.04955(11)$ & $0.29062(14)$ & $0.0287(6)$ \\
\hline $\mathrm{H} 27$ & 0.374654 & 0.061415 & 0.275938 & $0.034^{*}$ \\
\hline $\mathrm{C} 17$ & $0.4208(2)$ & $-0.00760(12)$ & $0.29044(17)$ & $0.0436(9)$ \\
\hline $\mathrm{H} 30$ & 0.412317 & -0.019769 & 0.244642 & $0.065^{*}$ \\
\hline $\mathrm{H} 28$ & 0.384677 & -0.019880 & 0.320468 & $0.065^{*}$ \\
\hline H29 & 0.465295 & -0.019921 & 0.306087 & $0.065^{*}$ \\
\hline $\mathrm{C} 18$ & $0.4739(2)$ & $0.06968(17)$ & $0.24131(17)$ & $0.0512(10)$ \\
\hline H32 & 0.460857 & 0.060380 & 0.195346 & $0.077 *$ \\
\hline H33 & 0.518819 & 0.055308 & 0.251868 & $0.077 *$ \\
\hline H31 & 0.476153 & 0.106295 & 0.245001 & $0.077^{*}$ \\
\hline C19 & $0.26615(17)$ & $0.00515(11)$ & $0.55876(15)$ & $0.0290(6)$ \\
\hline $\mathrm{C} 20$ & $0.23614(18)$ & $-0.02982(12)$ & $0.51569(17)$ & $0.0341(7)$ \\
\hline H34 & 0.213786 & -0.060076 & 0.528125 & $0.041^{*}$ \\
\hline $\mathrm{C} 21$ & $0.24547(15)$ & $-0.01156(11)$ & $0.45133(16)$ & $0.0268(7)$ \\
\hline $\mathrm{C} 22$ & $0.27416(19)$ & $0.00427(12)$ & $0.63403(15)$ & $0.0362(7)$ \\
\hline H35 & 0.283022 & 0.039493 & 0.648967 & $0.043^{*}$ \\
\hline $\mathrm{C} 23$ & $0.3355(2)$ & $-0.02675(15)$ & $0.65418(18)$ & $0.0469(9)$ \\
\hline H37 & 0.340200 & -0.026421 & 0.703283 & $0.070^{*}$ \\
\hline H38 & 0.376857 & -0.012635 & 0.633662 & $0.070^{*}$ \\
\hline H36 & 0.329249 & -0.061345 & 0.638663 & $0.070^{*}$ \\
\hline $\mathrm{C} 24$ & $0.2098(2)$ & $-0.01320(16)$ & $0.66876(19)$ & $0.0524(10)$ \\
\hline H39 & 0.217036 & -0.013151 & 0.717576 & $0.079 *$ \\
\hline H41 & 0.198657 & -0.047253 & 0.653800 & $0.079 *$ \\
\hline $\mathrm{H} 40$ & 0.172026 & 0.009446 & 0.657489 & $0.079^{*}$ \\
\hline $\mathrm{C} 25$ & $0.22093(18)$ & $-0.03350(12)$ & $0.38605(16)$ & $0.0346(7)$ \\
\hline H42 & 0.258186 & -0.029403 & 0.351965 & $0.041^{*}$ \\
\hline $\mathrm{C} 26$ & $0.2065(2)$ & $-0.08964(13)$ & $0.3940(2)$ & $0.0525(10)$ \\
\hline H45 & 0.194344 & -0.103937 & 0.350169 & $0.079 *$ \\
\hline H44 & 0.168549 & -0.094462 & 0.425556 & $0.079 *$ \\
\hline H43 & 0.247523 & -0.106445 & 0.411353 & $0.079^{*}$ \\
\hline $\mathrm{C} 27$ & $0.1578(2)$ & $-0.00645(14)$ & $0.36021(19)$ & $0.0471(9)$ \\
\hline H47 & 0.143055 & -0.021654 & 0.317693 & $0.071 *$ \\
\hline H46 & 0.168614 & 0.029013 & 0.352819 & $0.071 *$ \\
\hline H48 & 0.120884 & -0.009254 & 0.393429 & $0.071^{*}$ \\
\hline $\mathrm{C} 28$ & $0.17695(16)$ & $0.13410(11)$ & $0.67075(15)$ & $0.0291(6)$ \\
\hline $\mathrm{C} 29$ & $0.10972(16)$ & $0.11984(13)$ & $0.65687(15)$ & $0.0353(7)$ \\
\hline H49 & 0.071667 & 0.121593 & 0.686550 & $0.042 *$ \\
\hline C30 & $0.10960(17)$ & $0.10284(13)$ & $0.59193(16)$ & $0.0353(7)$ \\
\hline $\mathrm{C} 31$ & $0.20606(18)$ & $0.15603(13)$ & $0.73387(16)$ & $0.0373(8)$ \\
\hline $\mathrm{H} 50$ & 0.247595 & 0.136106 & 0.746193 & $0.045^{*}$ \\
\hline C32 & $0.2284(3)$ & $0.20987(16)$ & $0.7225(2)$ & $0.0698(13)$ \\
\hline H53 & 0.247710 & 0.223405 & 0.764241 & $0.105^{*}$ \\
\hline H51 & 0.188727 & 0.230049 & 0.709049 & $0.105^{*}$ \\
\hline H52 & 0.263059 & 0.210933 & 0.686909 & $0.105^{*}$ \\
\hline C33 & $0.1558(2)$ & $0.15271(18)$ & $0.79216(19)$ & $0.0608(11)$ \\
\hline
\end{tabular}




\begin{tabular}{|c|c|c|c|c|c|}
\hline H55 & 0.176796 & 0.167016 & 0.832654 & $0.091^{*}$ & \\
\hline H54 & 0.144132 & 0.117499 & 0.800382 & $0.091^{*}$ & \\
\hline H56 & 0.114222 & 0.171483 & 0.781002 & $0.091^{*}$ & \\
\hline C34A & $0.0541(2)$ & $0.08120(17)$ & $0.54784(19)$ & $0.0532(10)$ & $0.574(10)$ \\
\hline H57A & 0.013350 & 0.102080 & 0.559735 & $0.064 *$ & $0.574(10)$ \\
\hline C34B & $0.0541(2)$ & $0.08120(17)$ & $0.54784(19)$ & $0.0532(10)$ & $0.426(10)$ \\
\hline H57B & 0.066428 & 0.044801 & 0.548716 & $0.064 *$ & $0.426(10)$ \\
\hline $\mathrm{C} 35$ & $0.0648(2)$ & $0.09304(16)$ & $0.47511(18)$ & $0.0532(10)$ & \\
\hline H58 & 0.071183 & 0.129268 & 0.469644 & $0.080^{*}$ & \\
\hline H59 & 0.024658 & 0.082248 & 0.449277 & $0.080 *$ & \\
\hline H60 & 0.105487 & 0.075371 & 0.458703 & $0.080^{*}$ & \\
\hline C36A & $0.0340(5)$ & 0.0322 & $0.5685(5)$ & $0.076(3)$ & $0.574(10)$ \\
\hline H61A & 0.020303 & 0.033042 & 0.615986 & $0.114^{*}$ & $0.574(10)$ \\
\hline H62A & 0.072501 & 0.009055 & 0.562895 & $0.114 *$ & $0.574(10)$ \\
\hline H63A & -0.004663 & 0.020941 & 0.541006 & $0.114 *$ & $0.574(10)$ \\
\hline $\mathrm{C} 36 \mathrm{~B}$ & $-0.0104(5)$ & $0.0799(5)$ & $0.5750(5)$ & $0.063(4)$ & $0.426(10)$ \\
\hline H61B & -0.009018 & 0.062495 & 0.618433 & $0.095^{*}$ & $0.426(10)$ \\
\hline H62B & -0.041222 & 0.061930 & 0.544371 & $0.095 *$ & $0.426(10)$ \\
\hline H63B & -0.027094 & 0.114175 & 0.581517 & $0.095^{*}$ & $0.426(10)$ \\
\hline C37 & $0.43817(16)$ & $0.24760(12)$ & $0.54152(15)$ & $0.0315(7)$ & \\
\hline C38 & $0.46062(18)$ & 0.27479 (13) & $0.48529(16)$ & $0.0387(8)$ & \\
\hline H64 & 0.484238 & 0.305937 & 0.485591 & $0.046^{*}$ & \\
\hline C39 & $0.44185(17)$ & $0.24765(12)$ & $0.43003(15)$ & $0.0341(7)$ & \\
\hline $\mathrm{C} 40$ & $0.4448(2)$ & $0.26136(14)$ & $0.61417(16)$ & $0.0458(9)$ & \\
\hline H65 & 0.422813 & 0.234499 & 0.641828 & $0.055^{*}$ & \\
\hline C41 & $0.5196(3)$ & $0.2646(2)$ & $0.6334(2)$ & $0.0818(17)$ & \\
\hline H67 & 0.523519 & 0.275708 & 0.680245 & $0.123^{*}$ & \\
\hline $\mathrm{H} 68$ & 0.542805 & 0.288635 & 0.603906 & $0.123^{*}$ & \\
\hline H66 & 0.540852 & 0.231495 & 0.628500 & $0.123^{*}$ & \\
\hline C42 & $0.4073(3)$ & $0.3109(2)$ & $0.6274(2)$ & $0.0861(18)$ & \\
\hline H69 & 0.412874 & 0.320390 & 0.674707 & $0.129^{*}$ & \\
\hline $\mathrm{H} 71$ & 0.358544 & 0.306816 & 0.617231 & $0.129^{*}$ & \\
\hline $\mathrm{H} 70$ & 0.426594 & 0.337220 & 0.598575 & $0.129 *$ & \\
\hline C43A & $0.4504(2)$ & $0.25747(16)$ & $0.35604(17)$ & $0.0512(10)$ & $0.719(16)$ \\
\hline $\mathrm{H} 72 \mathrm{~A}$ & 0.437857 & 0.293647 & 0.351016 & $0.061^{*}$ & $0.719(16)$ \\
\hline C43B & $0.4504(2)$ & $0.25747(16)$ & $0.35604(17)$ & $0.0512(10)$ & $0.281(16)$ \\
\hline H72B & 0.470887 & 0.291775 & 0.353444 & $0.061^{*}$ & $0.281(16)$ \\
\hline C44A & 0.5189 (4) & $0.2553(5)$ & $0.3336(3)$ & $0.072(3)$ & $0.719(16)$ \\
\hline H73A & 0.521137 & 0.266047 & 0.286454 & $0.108^{*}$ & 0.719 (16) \\
\hline H74A & 0.535842 & 0.220884 & 0.337503 & $0.108^{*}$ & $0.719(16)$ \\
\hline $\mathrm{H} 75 \mathrm{~A}$ & 0.547159 & 0.277643 & 0.361229 & $0.108^{*}$ & $0.719(16)$ \\
\hline C44B & $0.5127(12)$ & $0.2193(12)$ & $0.3331(8)$ & $0.071(6)$ & $0.281(16)$ \\
\hline H73B & 0.551346 & 0.222943 & 0.364141 & $0.106^{*}$ & $0.281(16)$ \\
\hline H74B & 0.527507 & 0.227687 & 0.287342 & $0.106^{*}$ & $0.281(16)$ \\
\hline H75B & 0.496165 & 0.184595 & 0.334190 & $0.106^{*}$ & $0.281(16)$ \\
\hline $\mathrm{C} 45 \mathrm{~A}$ & $0.3975(4)$ & $0.2304(5)$ & $0.3138(3)$ & $0.057(2)$ & $0.719(16)$ \\
\hline H76A & 0.351629 & 0.237583 & 0.331277 & $0.085^{*}$ & $0.719(16)$ \\
\hline H77A & 0.405947 & 0.194154 & 0.315773 & $0.085^{*}$ & $0.719(16)$ \\
\hline
\end{tabular}




$\begin{array}{llllll}\text { H78A } & 0.400717 & 0.241788 & 0.267033 & 0.085^{*} & 0.719(16) \\ \text { C45B } & 0.3905(11) & 0.2610(10) & 0.3212(9) & 0.058(5) & 0.281(16) \\ \text { H76B } & 0.400386 & 0.264898 & 0.273114 & 0.087^{*} & 0.281(16) \\ \text { H77B } & 0.364638 & 0.290208 & 0.337140 & 0.087^{*} & 0.281(16) \\ \text { H78B } & 0.363373 & 0.230557 & 0.328252 & 0.087^{*} & 0.281(16) \\ \text { B } & 0.30572(17) & 0.06863(11) & 0.40118(16) & 0.0217(6) & \\ \text { H1 } & 0.295416 & 0.053563 & 0.356212 & 0.026^{*} & \\ \text { N1 } & 0.26882(12) & 0.12017(8) & 0.40630(10) & 0.0207(4) & \\ \text { N2 } & 0.27389(12) & 0.14851(8) & 0.46446(11) & 0.0224(5) & \\ \text { N3 } & 0.38405(12) & 0.07583(8) & 0.40739(11) & 0.0217(5) & \\ \text { N4 } & 0.41373(12) & 0.09024(9) & 0.46732(11) & 0.0234(5) & \\ \text { N5 } & 0.27948(12) & 0.03263(8) & 0.45666(11) & 0.0223(5) & \\ \text { N6 } & 0.29237(12) & 0.04292(9) & 0.52350(11) & 0.0250(5) & \\ \text { N7 } & 0.17398(13) & 0.10721(9) & 0.57009(13) & 0.0310(5) & \\ \text { H2 } & 0.187129 & 0.098662 & 0.529310 & 0.037^{*} & \\ \text { N8 } & 0.21714(13) & 0.12622(9) & 0.61732(12) & 0.0298(5) & \\ \text { N9 } & 0.41090(14) & 0.20651(10) & 0.45416(12) & 0.0306(6) & \\ \text { H3 } & 0.394258 & 0.182617 & 0.428346 & 0.037^{*} & \\ \text { N10 } & 0.40781(13) & 0.20540(10) & 0.52242(12) & 0.0289(5) & \\ \text { I } & 0.42224(2) & 0.11967(2) & 0.71250(2) & 0.04898(7) & \\ \text { EU } & 0.34693(2) & 0.12375(2) & 0.57077(2) & 0.02286(5) & \end{array}$

Atomic displacement parameters $\left(\AA^{2}\right)$

\begin{tabular}{|c|c|c|c|c|c|c|}
\hline & $U^{11}$ & $U^{22}$ & $U^{33}$ & $U^{12}$ & $U^{13}$ & $U^{23}$ \\
\hline $\mathrm{C} 1$ & $0.0221(14)$ & $0.0230(14)$ & 0.0245 (14) & $0.0014(12)$ & 0.0007 (11) & $-0.0010(11)$ \\
\hline $\mathrm{C} 2$ & $0.0259(16)$ & $0.0254(14)$ & $0.0305(15)$ & $0.0057(12)$ & $-0.0045(12)$ & $0.0012(11)$ \\
\hline $\mathrm{C} 3$ & $0.0214(14)$ & $0.0251(13)$ & $0.0248(14)$ & $0.0008(12)$ & $-0.0002(11)$ & 0.0017 (11) \\
\hline $\mathrm{C} 4$ & $0.0319(16)$ & $0.0263(14)$ & $0.0268(15)$ & $0.0066(13)$ & $-0.0006(13)$ & $-0.0028(12)$ \\
\hline $\mathrm{C} 5$ & $0.044(2)$ & $0.0286(16)$ & $0.043(2)$ & $-0.0008(15)$ & $0.0017(15)$ & $-0.0063(14)$ \\
\hline C6 & $0.041(2)$ & $0.0350(17)$ & $0.0383(18)$ & $0.0086(15)$ & $0.0065(15)$ & $-0.0033(13)$ \\
\hline $\mathrm{C} 7$ & $0.0332(16)$ & $0.0271(14)$ & 0.0269 (14) & $0.0015(13)$ & $-0.0069(12)$ & $-0.0031(12)$ \\
\hline $\mathrm{C} 8$ & $0.042(2)$ & $0.063(2)$ & $0.043(2)$ & 0.0119 (19) & $-0.0176(16)$ & $-0.0166(17)$ \\
\hline C9 & $0.055(2)$ & $0.0339(17)$ & $0.0287(16)$ & $0.0040(17)$ & $0.0029(16)$ & $-0.0004(13)$ \\
\hline $\mathrm{C} 10$ & $0.0183(14)$ & $0.0261(14)$ & 0.0288 (14) & $-0.0018(12)$ & $0.0004(11)$ & $0.0004(11)$ \\
\hline $\mathrm{C} 11$ & $0.0165(14)$ & 0.0387 (17) & $0.0270(15)$ & 0.0004 (13) & $0.0028(11)$ & $-0.0005(12)$ \\
\hline C12 & $0.0208(15)$ & $0.0276(14)$ & 0.0229 (13) & $0.0021(12)$ & $0.0041(11)$ & $0.0026(11)$ \\
\hline C13 & $0.0218(15)$ & $0.0392(16)$ & $0.0316(16)$ & $-0.0052(13)$ & $-0.0003(12)$ & $-0.0060(13)$ \\
\hline $\mathrm{C} 14$ & $0.042(2)$ & $0.050(2)$ & 0.0380 (19) & $-0.0024(17)$ & $-0.0161(15)$ & $0.0061(15)$ \\
\hline $\mathrm{C} 15$ & $0.037(2)$ & $0.049(2)$ & $0.059(2)$ & $-0.0175(17)$ & $-0.0167(17)$ & $0.0002(17)$ \\
\hline $\mathrm{C} 16$ & $0.0233(15)$ & $0.0394(16)$ & $0.0235(14)$ & $0.0020(13)$ & $0.0004(12)$ & $0.0018(12)$ \\
\hline $\mathrm{C} 17$ & $0.056(2)$ & $0.0411(18)$ & $0.0332(17)$ & $0.0113(17)$ & $-0.0097(16)$ & $-0.0104(14)$ \\
\hline C18 & $0.042(2)$ & $0.085(3)$ & $0.0259(17)$ & $-0.007(2)$ & $0.0069(15)$ & 0.0005 (17) \\
\hline C19 & $0.0272(15)$ & $0.0295(15)$ & $0.0304(16)$ & $0.0003(13)$ & $0.0055(13)$ & 0.0063 (12) \\
\hline $\mathrm{C} 20$ & $0.0340(17)$ & $0.0284(16)$ & $0.0398(18)$ & $-0.0063(14)$ & $0.0072(15)$ & $0.0070(13)$ \\
\hline $\mathrm{C} 21$ & $0.0226(16)$ & $0.0230(15)$ & $0.0348(17)$ & $-0.0024(12)$ & $0.0034(11)$ & $0.0012(12)$ \\
\hline $\mathrm{C} 22$ & $0.043(2)$ & $0.0365(17)$ & $0.0291(16)$ & $0.0030(16)$ & $0.0084(14)$ & $0.0101(13)$ \\
\hline $\mathrm{C} 23$ & $0.045(2)$ & $0.059(2)$ & $0.0371(19)$ & 0.0125 (19) & $0.0034(16)$ & $0.0110(16)$ \\
\hline
\end{tabular}




\begin{tabular}{|c|c|c|c|c|c|c|}
\hline $\mathrm{C} 24$ & $0.045(2)$ & $0.072(3)$ & $0.041(2)$ & $0.005(2)$ & $0.0151(17)$ & $0.0221(18)$ \\
\hline $\mathrm{C} 25$ & $0.0351(19)$ & $0.0308(16)$ & $0.0378(17)$ & $-0.0121(14)$ & $0.0035(14)$ & $-0.0043(13)$ \\
\hline $\mathrm{C} 26$ & $0.068(3)$ & $0.0303(18)$ & $0.060(2)$ & $-0.0145(19)$ & $0.000(2)$ & $-0.0075(16)$ \\
\hline $\mathrm{C} 27$ & $0.044(2)$ & $0.048(2)$ & $0.050(2)$ & $-0.0111(18)$ & $-0.0107(17)$ & $-0.0014(16)$ \\
\hline $\mathrm{C} 28$ & $0.0284(15)$ & $0.0262(15)$ & $0.0328(15)$ & $0.0022(12)$ & $0.0073(12)$ & $0.0018(12)$ \\
\hline $\mathrm{C} 29$ & $0.0278(16)$ & $0.0426(18)$ & $0.0355(16)$ & $-0.0032(15)$ & $0.0066(13)$ & $0.0021(14)$ \\
\hline C 30 & $0.0268(17)$ & $0.0393(17)$ & $0.0398(17)$ & $-0.0073(14)$ & $0.0024(13)$ & $0.0068(14)$ \\
\hline $\mathrm{C} 31$ & $0.0373(19)$ & $0.0404(18)$ & $0.0342(17)$ & $0.0038(15)$ & $0.0001(14)$ & $-0.0040(14)$ \\
\hline $\mathrm{C} 32$ & $0.098(4)$ & $0.056(3)$ & $0.055(3)$ & $-0.024(3)$ & $-0.013(3)$ & $-0.012(2)$ \\
\hline $\mathrm{C} 33$ & 0.060 & $0.086(3)$ & $0.037(2)$ & $-0.006(2)$ & $0.0109(19)$ & $-0.018(2)$ \\
\hline C34A & $0.039(2)$ & $0.073(3)$ & $0.047(2)$ & $-0.025(2)$ & $-0.0084(17)$ & $0.0071(19)$ \\
\hline C34B & $0.039(2)$ & $0.073(3)$ & $0.047(2)$ & $-0.025(2)$ & $-0.0084(17)$ & $0.0071(19)$ \\
\hline C35 & $0.048(2)$ & $0.067(3)$ & $0.045(2)$ & $-0.006(2)$ & -0.0090 & $-0.0075(18)$ \\
\hline C36A & $0.070(6)$ & $0.064(5)$ & $0.095(6)$ & $-0.041(5)$ & $-0.037(5)$ & $0.019(5)$ \\
\hline C36B & $0.025(5)$ & $0.103(9)$ & $0.061(6)$ & $-0.020(5)$ & $0.011(4)$ & $-0.009(6)$ \\
\hline $\mathrm{C} 37$ & $0.0315(17)$ & $0.0348(16)$ & $0.0281(15)$ & $-0.0101(14)$ & $0.0032(13)$ & $-0.0003(12)$ \\
\hline C38 & $0.045(2)$ & $0.0397(18)$ & $0.0311(16)$ & $-0.0199(16)$ & $0.0014(15)$ & $0.0022(13)$ \\
\hline C39 & $0.0349(17)$ & $0.0413(17)$ & $0.0261(15)$ & $-0.0099(15)$ & $0.0018(14)$ & $0.0075(14)$ \\
\hline $\mathrm{C} 40$ & $0.055(2)$ & $0.057(2)$ & $0.0251(16)$ & $-0.0231(19)$ & $0.0060(15)$ & $-0.0063(15)$ \\
\hline $\mathrm{C} 41$ & $0.072(3)$ & $0.141(5)$ & $0.033(2)$ & $-0.041(3)$ & $-0.014(2)$ & $0.003(3)$ \\
\hline $\mathrm{C} 42$ & $0.125(5)$ & $0.085(4)$ & $0.048(3)$ & $-0.001(3)$ & $0.022(3)$ & $-0.027(2)$ \\
\hline $\mathrm{C} 43 \mathrm{~A}$ & $0.064(3)$ & $0.065(2)$ & $0.0245(16)$ & $-0.022(2)$ & $0.0027(17)$ & $0.0075(16)$ \\
\hline $\mathrm{C} 43 \mathrm{~B}$ & $0.064(3)$ & $0.065(2)$ & $0.0245(16)$ & $-0.022(2)$ & $0.0027(17)$ & $0.0075(16)$ \\
\hline $\mathrm{C} 44 \mathrm{~A}$ & $0.058(4)$ & $0.128(8)$ & $0.031(3)$ & $-0.041(5)$ & $0.004(3)$ & $0.004(4)$ \\
\hline C44B & $0.074(11)$ & $0.118(14)$ & $0.021(6)$ & $0.022(11)$ & $0.018(7)$ & $-0.003(9)$ \\
\hline $\mathrm{C} 45 \mathrm{~A}$ & $0.054(4)$ & $0.093(6)$ & $0.023(3)$ & $-0.027(4)$ & $-0.005(2)$ & $0.007(3)$ \\
\hline $\mathrm{C} 45 \mathrm{~B}$ & $0.081(11)$ & $0.062(11)$ & $0.031(7)$ & $-0.019(10)$ & $-0.007(7)$ & $0.017(8)$ \\
\hline $\mathrm{B}$ & $0.0184(16)$ & $0.0228(15)$ & $0.0239(15)$ & $-0.0031(13)$ & $-0.0004(12)$ & $-0.0019(12)$ \\
\hline N1 & $0.0199(11)$ & $0.0201(11)$ & $0.0222(10)$ & $0.0001(10)$ & $0.0009(9)$ & $-0.0001(9)$ \\
\hline $\mathrm{N} 2$ & $0.0226(12)$ & $0.0242(12)$ & $0.0204(11)$ & $-0.0020(10)$ & $0.0010(9)$ & $-0.0024(9)$ \\
\hline N3 & $0.0205(12)$ & $0.0241(11)$ & $0.0204(11)$ & $-0.0007(10)$ & $0.0002(9)$ & $0.0006(9)$ \\
\hline N4 & $0.0180(12)$ & $0.0269(12)$ & $0.0252(12)$ & $-0.0016(10)$ & $-0.0020(9)$ & $-0.0040(9)$ \\
\hline N5 & $0.0189(12)$ & $0.0236(11)$ & $0.0245(11)$ & $-0.0014(10)$ & $0.0008(9)$ & $0.0005(9)$ \\
\hline N6 & $0.0231(13)$ & $0.0297(12)$ & $0.0222(11)$ & $-0.0009(10)$ & $0.0028(9)$ & $0.0022(9)$ \\
\hline N7 & $0.0296(14)$ & $0.0359(13)$ & $0.0274(12)$ & $-0.0050(11)$ & $0.0033(11)$ & $-0.0035(11)$ \\
\hline N8 & $0.0286(13)$ & $0.0304(13)$ & $0.0303(12)$ & $-0.0030(12)$ & $0.0006(10)$ & $-0.0002(11)$ \\
\hline N9 & $0.0353(15)$ & $0.0348(14)$ & $0.0216(11)$ & $-0.0072(12)$ & $-0.0006(11)$ & $-0.0016(10)$ \\
\hline N10 & $0.0300(14)$ & $0.0361(14)$ & $0.0206(11)$ & $-0.0020(11)$ & $-0.0001(10)$ & $0.0026(10)$ \\
\hline I & $0.04194(13)$ & $0.07629(18)$ & $0.02871(11)$ & $0.00290(13)$ & $-0.00875(9)$ & $0.00910(11)$ \\
\hline EU & $0.02157(7)$ & $0.02746(7)$ & $0.01955(7)$ & $-0.00247(6)$ & $0.00070(5)$ & $0.00068(6)$ \\
\hline
\end{tabular}

Geometric parameters $\left(\AA,{ }^{\circ}\right)$

\begin{tabular}{llll}
\hline $\mathrm{C} 1-\mathrm{N} 2$ & $1.331(4)$ & $\mathrm{C} 30-\mathrm{N} 7$ & $1.336(4)$ \\
$\mathrm{C} 1-\mathrm{C} 2$ & $1.393(4)$ & $\mathrm{C} 30-\mathrm{C} 34 \mathrm{~A}$ & $1.508(5)$ \\
$\mathrm{C} 1-\mathrm{C} 4$ & $1.504(4)$ & $\mathrm{C} 30-\mathrm{C} 34 \mathrm{~B}$ & $1.508(5)$ \\
$\mathrm{C} 2-\mathrm{C} 3$ & $1.379(4)$ & $\mathrm{C} 31-\mathrm{C} 32$ & $1.517(5)$ \\
$\mathrm{C} 2-\mathrm{H} 4$ & 0.9500 & $\mathrm{C} 31-\mathrm{C} 33$ & $1.520(5)$
\end{tabular}




\begin{tabular}{|c|c|c|c|}
\hline $\mathrm{C} 3-\mathrm{N} 1$ & $1.360(3)$ & C31-H50 & 1.0000 \\
\hline $\mathrm{C} 3-\mathrm{C} 7$ & $1.501(4)$ & C32-H53 & 0.9800 \\
\hline $\mathrm{C} 4-\mathrm{C} 6$ & $1.527(4)$ & $\mathrm{C} 32-\mathrm{H} 51$ & 0.9800 \\
\hline $\mathrm{C} 4-\mathrm{C} 5$ & $1.529(4)$ & C32- & 0.9800 \\
\hline $\mathrm{C} 4-\mathrm{H} 5$ & 1.0000 & C33- & 0.9800 \\
\hline $\mathrm{C} 5-\mathrm{H} 8$ & 0.9800 & C33-H54 & 0.9800 \\
\hline $\mathrm{C} 5-\mathrm{H} 6$ & 0.9800 & C33-H56 & 0.9800 \\
\hline $\mathrm{C} 5-\mathrm{H} 7$ & 0.9800 & $\mathrm{C} 34 \mathrm{a}-\mathrm{C} 36 \mathrm{~A}$ & $1.424(8)$ \\
\hline $\mathrm{C} 6-\mathrm{H} 10$ & 0.9800 & $\mathrm{C} 34 \mathrm{a}-\mathrm{C} 35$ & $1.494(5)$ \\
\hline C6- $-\mathrm{H} 9$ & 0.9800 & $\mathrm{C} 34 \mathrm{a}-\mathrm{H} 57 \mathrm{~A}$ & 1.0000 \\
\hline C6-H11 & 0.9800 & $\mathrm{C} 34 \mathrm{~b}-\mathrm{C} 36 \mathrm{~B}$ & $1.371(9)$ \\
\hline $\mathrm{C} 7-\mathrm{C} 9$ & $1.521(4)$ & $\mathrm{C} 34 \mathrm{~b}-\mathrm{C} 35$ & $1.494(5)$ \\
\hline $\mathrm{C} 7-\mathrm{C} 8$ & $1.531(4)$ & $\mathrm{C} 34 \mathrm{~b}-\mathrm{H} 57 \mathrm{~B}$ & 1.0000 \\
\hline $\mathrm{C} 7-\mathrm{H} 12$ & 1.0000 & C35-H58 & 0.9800 \\
\hline $\mathrm{C} 8-\mathrm{H} 14$ & 0.9800 & C35-H59 & 0.9800 \\
\hline $\mathrm{C} 8-\mathrm{H} 13$ & 0.9800 & $\mathrm{C} 35-\mathrm{H} 60$ & 0.9800 \\
\hline $\mathrm{C} 8-\mathrm{H} 15$ & 0.9800 & $\mathrm{C} 36 \mathrm{a}-\mathrm{H} 61 \mathrm{~A}$ & 0.9800 \\
\hline C9- $-\mathrm{H} 17$ & 0.9800 & $\mathrm{C} 36 \mathrm{a}-\mathrm{H} 62 \mathrm{~A}$ & 0.9800 \\
\hline C9-H18 & 0.9800 & $\mathrm{C} 36 \mathrm{a}-\mathrm{H} 63 \mathrm{~A}$ & 0.9800 \\
\hline C9-H16 & 0.9800 & $\mathrm{C} 36 \mathrm{~b}-\mathrm{H} 61 \mathrm{~B}$ & 0.9800 \\
\hline $\mathrm{C} 10-\mathrm{N} 4$ & $1.334(4)$ & $\mathrm{C} 36 \mathrm{~b}-\mathrm{H} 62 \mathrm{~B}$ & 0.9800 \\
\hline $\mathrm{C} 10-\mathrm{C} 11$ & $1.387(4)$ & $\mathrm{C} 36 \mathrm{~b}-\mathrm{H} 63 \mathrm{~B}$ & 0.9800 \\
\hline $\mathrm{C} 10-\mathrm{C} 13$ & $1.502(4)$ & $\mathrm{C} 37-\mathrm{N} 10$ & $1.327(4)$ \\
\hline $\mathrm{C} 11-\mathrm{C} 12$ & $1.372(4)$ & $\mathrm{C} 37-\mathrm{C} 38$ & $1.402(4)$ \\
\hline C11-H19 & 0.9500 & $\mathrm{C} 37-\mathrm{C} 40$ & $1.495(4)$ \\
\hline $\mathrm{C} 12-\mathrm{N} 3$ & $1.360(4)$ & $\mathrm{C} 38-\mathrm{C} 39$ & $1.365(4)$ \\
\hline $\mathrm{C} 12-\mathrm{C} 16$ & $1.501(4)$ & C38-H64 & 0.9500 \\
\hline $\mathrm{C} 13-\mathrm{C} 15$ & $1.516(5)$ & $\mathrm{C} 39-\mathrm{N} 9$ & $1.341(4)$ \\
\hline $\mathrm{C} 13-\mathrm{C} 14$ & $1.523(5)$ & $\mathrm{C} 39-\mathrm{C} 43 \mathrm{~A}$ & $1.502(4)$ \\
\hline $\mathrm{C} 13-\mathrm{H} 20$ & 1.0000 & $\mathrm{C} 39-\mathrm{C} 43 \mathrm{~B}$ & $1.502(4)$ \\
\hline $\mathrm{C} 14-\mathrm{H} 21$ & 0.9800 & $\mathrm{C} 40-\mathrm{C} 41$ & $1.511(6)$ \\
\hline $\mathrm{C} 14-\mathrm{H} 22$ & 0.9800 & $\mathrm{C} 40-\mathrm{C} 42$ & $1.534(6)$ \\
\hline $\mathrm{C} 14-\mathrm{H} 23$ & 0.9800 & $\mathrm{C} 40-\mathrm{H} 65$ & 1.0000 \\
\hline $\mathrm{C} 15-\mathrm{H} 24$ & 0.9800 & $\mathrm{C} 41-\mathrm{H} 67$ & 0.9800 \\
\hline $\mathrm{C} 15-\mathrm{H} 25$ & 0.9800 & $\mathrm{C} 41-\mathrm{H} 68$ & 0.9800 \\
\hline $\mathrm{C} 15-\mathrm{H} 26$ & 0.9800 & $\mathrm{C} 41-\mathrm{H} 66$ & 0.9800 \\
\hline $\mathrm{C} 16-\mathrm{C} 18$ & $1.523(4)$ & $\mathrm{C} 42-\mathrm{H} 69$ & 0.9800 \\
\hline $\mathrm{C} 16-\mathrm{C} 17$ & $1.524(4)$ & $\mathrm{C} 42-\mathrm{H} 71$ & 0.9800 \\
\hline $\mathrm{C} 16-\mathrm{H} 27$ & 1.0000 & $\mathrm{C} 42-\mathrm{H} 70$ & 0.9800 \\
\hline C17- $\mathrm{H} 30$ & 0.9800 & $\mathrm{C} 43 \mathrm{a}-\mathrm{C} 44 \mathrm{~A}$ & $1.411(8)$ \\
\hline $\mathrm{C} 17-\mathrm{H} 28$ & 0.9800 & $\mathrm{C} 43 \mathrm{a}-\mathrm{C} 45 \mathrm{~A}$ & $1.514(8)$ \\
\hline C17-H29 & 0.9800 & $\mathrm{C} 43 \mathrm{a}-\mathrm{H} 72 \mathrm{~A}$ & 1.0000 \\
\hline C18-H32 & 0.9800 & $\mathrm{C} 43 \mathrm{~b}-\mathrm{C} 45 \mathrm{~B}$ & $1.36(2)$ \\
\hline C18-H33 & 0.9800 & $\mathrm{C} 43 \mathrm{~b}-\mathrm{C} 44 \mathrm{~B}$ & $1.651(19)$ \\
\hline $\mathrm{C} 18-\mathrm{H} 31$ & 0.9800 & $\mathrm{C} 43 \mathrm{~b}-\mathrm{H} 72 \mathrm{~B}$ & 1.0000 \\
\hline C19-N6 & $1.329(4)$ & $\mathrm{C} 44 \mathrm{a}-\mathrm{H} 73 \mathrm{~A}$ & 0.9800 \\
\hline $\mathrm{C} 19-\mathrm{C} 20$ & $1.395(5)$ & $\mathrm{C} 44 \mathrm{a}-\mathrm{H} 74 \mathrm{~A}$ & 0.9800 \\
\hline $\mathrm{C} 19-\mathrm{C} 22$ & $1.504(4)$ & $\mathrm{C} 44 \mathrm{a}-\mathrm{H} 75 \mathrm{~A}$ & 0.9800 \\
\hline
\end{tabular}




\begin{tabular}{|c|c|c|c|}
\hline $\mathrm{C} 20-\mathrm{C} 21$ & $1.380(4)$ & $\mathrm{C} 44 \mathrm{~b}-\mathrm{H} 73 \mathrm{~B}$ & 0.9800 \\
\hline $\mathrm{C} 20-\mathrm{H} 34$ & 0.9500 & $\mathrm{C} 44 \mathrm{~b}-\mathrm{H} 74 \mathrm{~B}$ & 0.9800 \\
\hline $\mathrm{C} 21-\mathrm{N} 5$ & $1.357(4)$ & $\mathrm{C} 44 \mathrm{~b}-\mathrm{H} 75 \mathrm{~B}$ & 0.9800 \\
\hline $\mathrm{C} 21-\mathrm{C} 25$ & $1.501(4)$ & $\mathrm{C} 45 \mathrm{a}-\mathrm{H} 76 \mathrm{~A}$ & 0.9800 \\
\hline $\mathrm{C} 22-\mathrm{C} 24$ & $1.507(5)$ & $\mathrm{C} 45 \mathrm{a}-\mathrm{H} 77 \mathrm{~A}$ & 0.9800 \\
\hline $\mathrm{C} 22-\mathrm{C} 23$ & $1.510(5)$ & $\mathrm{C} 45 \mathrm{a}-\mathrm{H} 78 \mathrm{~A}$ & 0.9800 \\
\hline $\mathrm{C} 22-\mathrm{H} 35$ & 1.0000 & $\mathrm{C} 45 \mathrm{~b}-\mathrm{H} 76 \mathrm{~B}$ & 0.9800 \\
\hline $\mathrm{C} 23-\mathrm{H} 37$ & 0.9800 & $\mathrm{C} 45 \mathrm{~b}-\mathrm{H} 77 \mathrm{~B}$ & 0.9800 \\
\hline C23-H38 & 0.9800 & $\mathrm{C} 45 \mathrm{~b}-\mathrm{H} 78 \mathrm{~B}$ & 0.9800 \\
\hline $\mathrm{C} 23-\mathrm{H} 36$ & 0.9800 & $\mathrm{~B}-\mathrm{N} 3$ & $1.547(4)$ \\
\hline $\mathrm{C} 24-\mathrm{H} 39$ & 0.9800 & $\mathrm{~B}-\mathrm{N} 5$ & $1.549(4)$ \\
\hline $\mathrm{C} 24-\mathrm{H} 41$ & 0.9800 & $\mathrm{~B}-\mathrm{N} 1$ & $1.555(4)$ \\
\hline $\mathrm{C} 24-\mathrm{H} 40$ & 0.9800 & $\mathrm{~B}-\mathrm{H} 1$ & 1.0000 \\
\hline $\mathrm{C} 25-\mathrm{C} 27$ & $1.518(5)$ & $\mathrm{N} 1-\mathrm{N} 2$ & $1.384(3)$ \\
\hline $\mathrm{C} 25-\mathrm{C} 26$ & $1.531(4)$ & $\mathrm{N} 2-\mathrm{EU}$ & $2.633(2)$ \\
\hline $\mathrm{C} 25-\mathrm{H} 42$ & 1.0000 & $\mathrm{~N} 3-\mathrm{N} 4$ & $1.379(3)$ \\
\hline $\mathrm{C} 26-\mathrm{H} 45$ & 0.9800 & $\mathrm{~N} 4-\mathrm{EU}$ & $2.593(2)$ \\
\hline $\mathrm{C} 26-\mathrm{H} 44$ & 0.9800 & N5-N6 & $1.379(3)$ \\
\hline $\mathrm{C} 26-\mathrm{H} 43$ & 0.9800 & N6-EU & $2.581(2)$ \\
\hline $\mathrm{C} 27-\mathrm{H} 47$ & 0.9800 & $\mathrm{~N} 7-\mathrm{N} 8$ & $1.359(3)$ \\
\hline $\mathrm{C} 27-\mathrm{H} 46$ & 0.9800 & $\mathrm{~N} 7-\mathrm{H} 2$ & 0.8800 \\
\hline $\mathrm{C} 27-\mathrm{H} 48$ & 0.9800 & $\mathrm{~N} 8-\mathrm{EU}$ & $2.699(2)$ \\
\hline $\mathrm{C} 28-\mathrm{N} 8$ & $1.337(4)$ & N9-N10 & $1.358(3)$ \\
\hline $\mathrm{C} 28-\mathrm{C} 29$ & $1.395(4)$ & N9- $\mathrm{H} 3$ & 0.8800 \\
\hline $\mathrm{C} 28-\mathrm{C} 31$ & $1.496(4)$ & $\mathrm{N} 10-\mathrm{EU}$ & $2.660(2)$ \\
\hline $\mathrm{C} 29-\mathrm{C} 30$ & $1.367(4)$ & $\mathrm{I}-\mathrm{EU}$ & $3.1788(2)$ \\
\hline $\mathrm{C} 29-\mathrm{H} 49$ & 0.9500 & & \\
\hline $\mathrm{N} 2-\mathrm{C} 1-\mathrm{C} 2$ & $110.6(2)$ & $\mathrm{C} 31-\mathrm{C} 32-\mathrm{H} 52$ & 109.5 \\
\hline $\mathrm{N} 2-\mathrm{C} 1-\mathrm{C} 4$ & $121.3(3)$ & $\mathrm{H} 53-\mathrm{C} 32-\mathrm{H} 52$ & 109.5 \\
\hline $\mathrm{C} 2-\mathrm{C} 1-\mathrm{C} 4$ & $128.1(3)$ & $\mathrm{H} 51-\mathrm{C} 32-\mathrm{H} 52$ & 109.5 \\
\hline $\mathrm{C} 3-\mathrm{C} 2-\mathrm{C} 1$ & $105.7(3)$ & $\mathrm{C} 31-\mathrm{C} 33-\mathrm{H} 55$ & 109.5 \\
\hline $\mathrm{C} 3-\mathrm{C} 2-\mathrm{H} 4$ & 127.1 & $\mathrm{C} 31-\mathrm{C} 33-\mathrm{H} 54$ & 109.5 \\
\hline $\mathrm{C} 1-\mathrm{C} 2-\mathrm{H} 4$ & 127.1 & $\mathrm{H} 55-\mathrm{C} 33-\mathrm{H} 54$ & 109.5 \\
\hline $\mathrm{N} 1-\mathrm{C} 3-\mathrm{C} 2$ & $108.0(2)$ & $\mathrm{C} 31-\mathrm{C} 33-\mathrm{H} 56$ & 109.5 \\
\hline $\mathrm{N} 1-\mathrm{C} 3-\mathrm{C} 7$ & $124.4(2)$ & $\mathrm{H} 55-\mathrm{C} 33-\mathrm{H} 56$ & 109.5 \\
\hline $\mathrm{C} 2-\mathrm{C} 3-\mathrm{C} 7$ & $127.6(3)$ & $\mathrm{H} 54-\mathrm{C} 33-\mathrm{H} 56$ & 109.5 \\
\hline $\mathrm{C} 1-\mathrm{C} 4-\mathrm{C} 6$ & $111.5(3)$ & $\mathrm{C} 36 \mathrm{a}-\mathrm{C} 34 \mathrm{a}-\mathrm{C} 35$ & $120.8(5)$ \\
\hline $\mathrm{C} 1-\mathrm{C} 4-\mathrm{C} 5$ & $110.9(3)$ & $\mathrm{C} 36 \mathrm{a}-\mathrm{C} 34 \mathrm{a}-\mathrm{C} 30$ & $112.4(4)$ \\
\hline $\mathrm{C} 6-\mathrm{C} 4-\mathrm{C} 5$ & $110.3(3)$ & $\mathrm{C} 35-\mathrm{C} 34 \mathrm{a}-\mathrm{C} 30$ & $112.4(3)$ \\
\hline $\mathrm{C} 1-\mathrm{C} 4-\mathrm{H} 5$ & 108.0 & $\mathrm{C} 36 \mathrm{a}-\mathrm{C} 34 \mathrm{a}-\mathrm{H} 57 \mathrm{~A}$ & 102.8 \\
\hline $\mathrm{C} 6-\mathrm{C} 4-\mathrm{H} 5$ & 108.0 & $\mathrm{C} 35-\mathrm{C} 34 \mathrm{a}-\mathrm{H} 57 \mathrm{~A}$ & 102.8 \\
\hline $\mathrm{C} 5-\mathrm{C} 4-\mathrm{H} 5$ & 108.0 & $\mathrm{C} 30-\mathrm{C} 34 \mathrm{a}-\mathrm{H} 57 \mathrm{~A}$ & 102.8 \\
\hline $\mathrm{C} 4-\mathrm{C} 5-\mathrm{H} 8$ & 109.5 & $\mathrm{C} 36 \mathrm{~b}-\mathrm{C} 34 \mathrm{~b}-\mathrm{C} 35$ & $120.9(6)$ \\
\hline $\mathrm{C} 4-\mathrm{C} 5-\mathrm{H} 6$ & 109.5 & $\mathrm{C} 36 \mathrm{~b}-\mathrm{C} 34 \mathrm{~b}-\mathrm{C} 30$ & $116.2(5)$ \\
\hline $\mathrm{H} 8-\mathrm{C} 5-\mathrm{H} 6$ & 109.5 & $\mathrm{C} 35-\mathrm{C} 34 \mathrm{~b}-\mathrm{C} 30$ & $112.4(3)$ \\
\hline $\mathrm{C} 4-\mathrm{C} 5-\mathrm{H} 7$ & 109.5 & $\mathrm{C} 36 \mathrm{~b}-\mathrm{C} 34 \mathrm{~b}-\mathrm{H} 57 \mathrm{~B}$ & 100.9 \\
\hline $\mathrm{H} 8-\mathrm{C} 5-\mathrm{H} 7$ & 109.5 & $\mathrm{C} 35-\mathrm{C} 34 \mathrm{~b}-\mathrm{H} 57 \mathrm{~B}$ & 100.9 \\
\hline
\end{tabular}




\begin{tabular}{|c|c|}
\hline $\mathrm{H} 6-\mathrm{C} 5-\mathrm{H} 7$ & 109.5 \\
\hline $\mathrm{C} 4-\mathrm{C} 6-\mathrm{H} 10$ & 109.5 \\
\hline $\mathrm{C} 4-\mathrm{C} 6-\mathrm{H} 9$ & 109.5 \\
\hline $\mathrm{H} 10-\mathrm{C} 6-\mathrm{H} 9$ & 109.5 \\
\hline $\mathrm{C} 4-\mathrm{C} 6-\mathrm{H} 11$ & 109.5 \\
\hline $\mathrm{H} 10-\mathrm{C} 6-\mathrm{H} 11$ & 109.5 \\
\hline $\mathrm{H} 9-\mathrm{C} 6-\mathrm{H} 11$ & 109.5 \\
\hline $\mathrm{C} 3-\mathrm{C} 7-\mathrm{C} 9$ & $110.6(2)$ \\
\hline $\mathrm{C} 3-\mathrm{C} 7-\mathrm{C} 8$ & $110.5(3)$ \\
\hline $\mathrm{C} 9-\mathrm{C} 7-\mathrm{C} 8$ & $110.5(3)$ \\
\hline $\mathrm{C} 3-\mathrm{C} 7-\mathrm{H} 12$ & 108.4 \\
\hline $\mathrm{C} 9-\mathrm{C} 7-\mathrm{H} 12$ & 108.4 \\
\hline $\mathrm{C} 8-\mathrm{C} 7-\mathrm{H} 12$ & 108.4 \\
\hline $\mathrm{C} 7-\mathrm{C} 8-\mathrm{H} 14$ & 109.5 \\
\hline $\mathrm{C} 7-\mathrm{C} 8-\mathrm{H} 13$ & 109.5 \\
\hline $\mathrm{H} 14-\mathrm{C} 8-\mathrm{H} 13$ & 109.5 \\
\hline $\mathrm{C} 7-\mathrm{C} 8-\mathrm{H} 15$ & 109.5 \\
\hline $\mathrm{H} 14-\mathrm{C} 8-\mathrm{H} 15$ & 109.5 \\
\hline $\mathrm{H} 13-\mathrm{C} 8-\mathrm{H} 15$ & 109.5 \\
\hline $\mathrm{C} 7-\mathrm{C} 9-\mathrm{H} 17$ & 109.5 \\
\hline $\mathrm{C} 7-\mathrm{C} 9-\mathrm{H} 18$ & 109.5 \\
\hline $\mathrm{H} 17-\mathrm{C} 9-\mathrm{H} 18$ & 109.5 \\
\hline C7-C9-H16 & 109.5 \\
\hline $\mathrm{H} 17-\mathrm{C} 9-\mathrm{H} 16$ & 109.5 \\
\hline $\mathrm{H} 18-\mathrm{C} 9-\mathrm{H} 16$ & 109.5 \\
\hline $\mathrm{N} 4-\mathrm{C} 10-\mathrm{C} 11$ & $110.0(2)$ \\
\hline $\mathrm{N} 4-\mathrm{C} 10-\mathrm{C} 13$ & $120.9(3)$ \\
\hline $\mathrm{C} 11-\mathrm{C} 10-\mathrm{C} 13$ & $129.0(3)$ \\
\hline $\mathrm{C} 12-\mathrm{C} 11-\mathrm{C} 10$ & $106.4(3)$ \\
\hline $\mathrm{C} 12-\mathrm{C} 11-\mathrm{H} 19$ & 126.8 \\
\hline $\mathrm{C} 10-\mathrm{C} 11-\mathrm{H} 19$ & 126.8 \\
\hline $\mathrm{N} 3-\mathrm{C} 12-\mathrm{C} 11$ & $107.6(2)$ \\
\hline $\mathrm{N} 3-\mathrm{C} 12-\mathrm{C} 16$ & $124.1(3)$ \\
\hline $\mathrm{C} 11-\mathrm{C} 12-\mathrm{C} 16$ & $128.2(3)$ \\
\hline $\mathrm{C} 10-\mathrm{C} 13-\mathrm{C} 15$ & $111.6(3)$ \\
\hline $\mathrm{C} 10-\mathrm{C} 13-\mathrm{C} 14$ & $110.7(3)$ \\
\hline $\mathrm{C} 15-\mathrm{C} 13-\mathrm{C} 14$ & $110.5(3)$ \\
\hline $\mathrm{C} 10-\mathrm{C} 13-\mathrm{H} 20$ & 107.9 \\
\hline $\mathrm{C} 15-\mathrm{C} 13-\mathrm{H} 20$ & 107.9 \\
\hline $\mathrm{C} 14-\mathrm{C} 13-\mathrm{H} 20$ & 107.9 \\
\hline $\mathrm{C} 13-\mathrm{C} 14-\mathrm{H} 21$ & 109.5 \\
\hline $\mathrm{C} 13-\mathrm{C} 14-\mathrm{H} 22$ & 109.5 \\
\hline $\mathrm{H} 21-\mathrm{C} 14-\mathrm{H} 22$ & 109.5 \\
\hline $\mathrm{C} 13-\mathrm{C} 14-\mathrm{H} 23$ & 109.5 \\
\hline $\mathrm{H} 21-\mathrm{C} 14-\mathrm{H} 23$ & 109.5 \\
\hline $\mathrm{H} 22-\mathrm{C} 14-\mathrm{H} 23$ & 109.5 \\
\hline $\mathrm{C} 13-\mathrm{C} 15-\mathrm{H} 24$ & 109.5 \\
\hline $\mathrm{C} 13-\mathrm{C} 15-\mathrm{H} 25$ & 109.5 \\
\hline
\end{tabular}

\begin{tabular}{|c|c|}
\hline $\mathrm{C} 30-\mathrm{C} 34 \mathrm{~b}-\mathrm{H} 57 \mathrm{~B}$ & 100.9 \\
\hline $\mathrm{C} 34 \mathrm{a}-\mathrm{C} 35-\mathrm{H} 58$ & 109.5 \\
\hline $\mathrm{C} 34 \mathrm{a}-\mathrm{C} 35-\mathrm{H} 59$ & 109.5 \\
\hline $\mathrm{H} 58-\mathrm{C} 35-\mathrm{H} 59$ & 109.5 \\
\hline $\mathrm{C} 34 \mathrm{a}-\mathrm{C} 35-\mathrm{H} 60$ & 109.5 \\
\hline $\mathrm{H} 58-\mathrm{C} 35-\mathrm{H} 60$ & 109.5 \\
\hline $\mathrm{H} 59-\mathrm{C} 35-\mathrm{H} 60$ & 109.5 \\
\hline $\mathrm{C} 34 \mathrm{a}-\mathrm{C} 36 \mathrm{a}-\mathrm{H} 61 \mathrm{~A}$ & 109.5 \\
\hline $\mathrm{C} 34 \mathrm{a}-\mathrm{C} 36 \mathrm{a}-\mathrm{H} 62 \mathrm{~A}$ & 109.5 \\
\hline $\mathrm{H} 61 \mathrm{a}-\mathrm{C} 36 \mathrm{a}-\mathrm{H} 62 \mathrm{~A}$ & 109.5 \\
\hline $\mathrm{C} 34 \mathrm{a}-\mathrm{C} 36 \mathrm{a}-\mathrm{H} 63 \mathrm{~A}$ & 109.5 \\
\hline $\mathrm{H} 61 \mathrm{a}-\mathrm{C} 36 \mathrm{a}-\mathrm{H} 63 \mathrm{~A}$ & 109.5 \\
\hline $\mathrm{H} 62 \mathrm{a}-\mathrm{C} 36 \mathrm{a}-\mathrm{H} 63 \mathrm{~A}$ & 109.5 \\
\hline $\mathrm{C} 34 \mathrm{~b}-\mathrm{C} 36 \mathrm{~b}-\mathrm{H} 61 \mathrm{~B}$ & 109.5 \\
\hline $\mathrm{C} 34 \mathrm{~b}-\mathrm{C} 36 \mathrm{~b}-\mathrm{H} 62 \mathrm{~B}$ & 109.5 \\
\hline $\mathrm{H} 61 \mathrm{~b}-\mathrm{C} 36 \mathrm{~b}-\mathrm{H} 62 \mathrm{~B}$ & 109.5 \\
\hline $\mathrm{C} 34 \mathrm{~b}-\mathrm{C} 36 \mathrm{~b}-\mathrm{H} 63 \mathrm{~B}$ & 109.5 \\
\hline $\mathrm{H} 61 \mathrm{~b}-\mathrm{C} 36 \mathrm{~b}-\mathrm{H} 63 \mathrm{~B}$ & 109.5 \\
\hline $\mathrm{H} 62 \mathrm{~b}-\mathrm{C} 36 \mathrm{~b}-\mathrm{H} 63 \mathrm{~B}$ & 109.5 \\
\hline $\mathrm{N} 10-\mathrm{C} 37-\mathrm{C} 38$ & $110.5(3)$ \\
\hline $\mathrm{N} 10-\mathrm{C} 37-\mathrm{C} 40$ & $121.5(3)$ \\
\hline $\mathrm{C} 38-\mathrm{C} 37-\mathrm{C} 40$ & $128.0(3)$ \\
\hline $\mathrm{C} 39-\mathrm{C} 38-\mathrm{C} 37$ & $106.4(3)$ \\
\hline C39-C38-H64 & 126.8 \\
\hline $\mathrm{C} 37-\mathrm{C} 38-\mathrm{H} 64$ & 126.8 \\
\hline N9-C39-C38 & $105.5(3)$ \\
\hline N9- C39-C43A & $122.8(3)$ \\
\hline $\mathrm{C} 38-\mathrm{C} 39-\mathrm{C} 43 \mathrm{~A}$ & $131.7(3)$ \\
\hline N9-C39-C43B & $122.8(3)$ \\
\hline $\mathrm{C} 38-\mathrm{C} 39-\mathrm{C} 43 \mathrm{~B}$ & $131.7(3)$ \\
\hline $\mathrm{C} 37-\mathrm{C} 40-\mathrm{C} 41$ & $110.0(3)$ \\
\hline $\mathrm{C} 37-\mathrm{C} 40-\mathrm{C} 42$ & $109.6(3)$ \\
\hline $\mathrm{C} 41-\mathrm{C} 40-\mathrm{C} 42$ & $111.7(4)$ \\
\hline $\mathrm{C} 37-\mathrm{C} 40-\mathrm{H} 65$ & 108.5 \\
\hline $\mathrm{C} 41-\mathrm{C} 40-\mathrm{H} 65$ & 108.5 \\
\hline $\mathrm{C} 42-\mathrm{C} 40-\mathrm{H} 65$ & 108.5 \\
\hline $\mathrm{C} 40-\mathrm{C} 41-\mathrm{H} 67$ & 109.5 \\
\hline $\mathrm{C} 40-\mathrm{C} 41-\mathrm{H} 68$ & 109.5 \\
\hline $\mathrm{H} 67-\mathrm{C} 41-\mathrm{H} 68$ & 109.5 \\
\hline $\mathrm{C} 40-\mathrm{C} 41-\mathrm{H} 66$ & 109.5 \\
\hline $\mathrm{H} 67-\mathrm{C} 41-\mathrm{H} 66$ & 109.5 \\
\hline $\mathrm{H} 68-\mathrm{C} 41-\mathrm{H} 66$ & 109.5 \\
\hline $\mathrm{C} 40-\mathrm{C} 42-\mathrm{H} 69$ & 109.5 \\
\hline $\mathrm{C} 40-\mathrm{C} 42-\mathrm{H} 71$ & 109.5 \\
\hline $\mathrm{H} 69-\mathrm{C} 42-\mathrm{H} 71$ & 109.5 \\
\hline $\mathrm{C} 40-\mathrm{C} 42-\mathrm{H} 70$ & 109.5 \\
\hline $\mathrm{H} 69-\mathrm{C} 42-\mathrm{H} 70$ & 109.5 \\
\hline $\mathrm{H} 71-\mathrm{C} 42-\mathrm{H} 70$ & 109.5 \\
\hline
\end{tabular}




\begin{tabular}{|c|c|}
\hline $\mathrm{H} 24-\mathrm{C} 15-\mathrm{H} 25$ & 109.5 \\
\hline $\mathrm{C} 13-\mathrm{C} 15-\mathrm{H} 26$ & 109.5 \\
\hline $\mathrm{H} 24-\mathrm{C} 15-\mathrm{H} 26$ & 109.5 \\
\hline $\mathrm{H} 25-\mathrm{C} 15-\mathrm{H} 26$ & 109.5 \\
\hline $\mathrm{C} 12-\mathrm{C} 16-\mathrm{C} 18$ & $111.1(3)$ \\
\hline $\mathrm{C} 12-\mathrm{C} 16-\mathrm{C} 17$ & $110.0(2)$ \\
\hline $\mathrm{C} 18-\mathrm{C} 16-\mathrm{C} 17$ & $110.6(3)$ \\
\hline $\mathrm{C} 12-\mathrm{C} 16-\mathrm{H} 27$ & 108.3 \\
\hline $\mathrm{C} 18-\mathrm{C} 16-\mathrm{H} 27$ & 108.3 \\
\hline $\mathrm{C} 17-\mathrm{C} 16-\mathrm{H} 27$ & 108.3 \\
\hline $\mathrm{C} 16-\mathrm{C} 17-\mathrm{H} 30$ & 109.5 \\
\hline $\mathrm{C} 16-\mathrm{C} 17-\mathrm{H} 28$ & 109.5 \\
\hline $\mathrm{H} 30-\mathrm{C} 17-\mathrm{H} 28$ & 109.5 \\
\hline $\mathrm{C} 16-\mathrm{C} 17-\mathrm{H} 29$ & 109.5 \\
\hline $\mathrm{H} 30-\mathrm{C} 17-\mathrm{H} 29$ & 109.5 \\
\hline $\mathrm{H} 28-\mathrm{C} 17-\mathrm{H} 29$ & 109.5 \\
\hline $\mathrm{C} 16-\mathrm{C} 18-\mathrm{H} 32$ & 109.5 \\
\hline $\mathrm{C} 16-\mathrm{C} 18-\mathrm{H} 33$ & 109.5 \\
\hline $\mathrm{H} 32-\mathrm{C} 18-\mathrm{H} 33$ & 109.5 \\
\hline $\mathrm{C} 16-\mathrm{C} 18-\mathrm{H} 31$ & 109.5 \\
\hline $\mathrm{H} 32-\mathrm{C} 18-\mathrm{H} 31$ & 109.5 \\
\hline $\mathrm{H} 33-\mathrm{C} 18-\mathrm{H} 31$ & 109.5 \\
\hline $\mathrm{N} 6-\mathrm{C} 19-\mathrm{C} 20$ & $110.2(3)$ \\
\hline $\mathrm{N} 6-\mathrm{C} 19-\mathrm{C} 22$ & $119.7(3)$ \\
\hline $\mathrm{C} 20-\mathrm{C} 19-\mathrm{C} 22$ & $130.1(3)$ \\
\hline $\mathrm{C} 21-\mathrm{C} 20-\mathrm{C} 19$ & $106.1(3)$ \\
\hline $\mathrm{C} 21-\mathrm{C} 20-\mathrm{H} 34$ & 127.0 \\
\hline $\mathrm{C} 19-\mathrm{C} 20-\mathrm{H} 34$ & 127.0 \\
\hline $\mathrm{N} 5-\mathrm{C} 21-\mathrm{C} 20$ & $107.4(3)$ \\
\hline $\mathrm{N} 5-\mathrm{C} 21-\mathrm{C} 25$ & $124.2(3)$ \\
\hline $\mathrm{C} 20-\mathrm{C} 21-\mathrm{C} 25$ & $128.4(3)$ \\
\hline $\mathrm{C} 19-\mathrm{C} 22-\mathrm{C} 24$ & $111.9(3)$ \\
\hline $\mathrm{C} 19-\mathrm{C} 22-\mathrm{C} 23$ & $110.8(3)$ \\
\hline $\mathrm{C} 24-\mathrm{C} 22-\mathrm{C} 23$ & $111.8(3)$ \\
\hline $\mathrm{C} 19-\mathrm{C} 22-\mathrm{H} 35$ & 107.4 \\
\hline $\mathrm{C} 24-\mathrm{C} 22-\mathrm{H} 35$ & 107.4 \\
\hline $\mathrm{C} 23-\mathrm{C} 22-\mathrm{H} 35$ & 107.4 \\
\hline $\mathrm{C} 22-\mathrm{C} 23-\mathrm{H} 37$ & 109.5 \\
\hline $\mathrm{C} 22-\mathrm{C} 23-\mathrm{H} 38$ & 109.5 \\
\hline $\mathrm{H} 37-\mathrm{C} 23-\mathrm{H} 38$ & 109.5 \\
\hline $\mathrm{C} 22-\mathrm{C} 23-\mathrm{H} 36$ & 109.5 \\
\hline $\mathrm{H} 37-\mathrm{C} 23-\mathrm{H} 36$ & 109.5 \\
\hline $\mathrm{H} 38-\mathrm{C} 23-\mathrm{H} 36$ & 109.5 \\
\hline $\mathrm{C} 22-\mathrm{C} 24-\mathrm{H} 39$ & 109.5 \\
\hline $\mathrm{C} 22-\mathrm{C} 24-\mathrm{H} 41$ & 109.5 \\
\hline $\mathrm{H} 39-\mathrm{C} 24-\mathrm{H} 41$ & 109.5 \\
\hline $\mathrm{C} 22-\mathrm{C} 24-\mathrm{H} 40$ & 109.5 \\
\hline $\mathrm{H} 39-\mathrm{C} 24-\mathrm{H} 40$ & 109.5 \\
\hline
\end{tabular}

\begin{tabular}{|c|c|}
\hline $\mathrm{C} 44 \mathrm{a}-\mathrm{C} 43 \mathrm{a}-\mathrm{C} 39$ & $114.0(4)$ \\
\hline $\mathrm{C} 44 \mathrm{a}-\mathrm{C} 43 \mathrm{a}-\mathrm{C} 45 \mathrm{~A}$ & $116.9(5)$ \\
\hline $\mathrm{C} 39-\mathrm{C} 43 \mathrm{a}-\mathrm{C} 45 \mathrm{~A}$ & $112.6(4)$ \\
\hline $\mathrm{C} 44 \mathrm{a}-\mathrm{C} 43 \mathrm{a}-\mathrm{H} 72 \mathrm{~A}$ & 103.8 \\
\hline $\mathrm{C} 39-\mathrm{C} 43 \mathrm{a}-\mathrm{H} 72 \mathrm{~A}$ & 103.8 \\
\hline $\mathrm{C} 45 \mathrm{a}-\mathrm{C} 43 \mathrm{a}-\mathrm{H} 72 \mathrm{~A}$ & 103.8 \\
\hline $\mathrm{C} 45 \mathrm{~b}-\mathrm{C} 43 \mathrm{~b}-\mathrm{C} 39$ & $114.5(8)$ \\
\hline $\mathrm{C} 45 \mathrm{~b}-\mathrm{C} 43 \mathrm{~b}-\mathrm{C} 44 \mathrm{~B}$ & $122.4(12)$ \\
\hline $\mathrm{C} 39-\mathrm{C} 43 \mathrm{~b}-\mathrm{C} 44 \mathrm{~B}$ & $104.1(7)$ \\
\hline $\mathrm{C} 45 \mathrm{~b}-\mathrm{C} 43 \mathrm{~b}-\mathrm{H} 72 \mathrm{~B}$ & 104.7 \\
\hline $\mathrm{C} 39-\mathrm{C} 43 \mathrm{~b}-\mathrm{H} 72 \mathrm{~B}$ & 104.7 \\
\hline $\mathrm{C} 44 \mathrm{~b}-\mathrm{C} 43 \mathrm{~b}-\mathrm{H} 72 \mathrm{~B}$ & 104.7 \\
\hline $\mathrm{C} 43 \mathrm{a}-\mathrm{C} 44 \mathrm{a}-\mathrm{H} 73 \mathrm{~A}$ & 109.5 \\
\hline $\mathrm{C} 43 \mathrm{a}-\mathrm{C} 44 \mathrm{a}-\mathrm{H} 74 \mathrm{~A}$ & 109.5 \\
\hline $\mathrm{H} 73 \mathrm{a}-\mathrm{C} 44 \mathrm{a}-\mathrm{H} 74 \mathrm{~A}$ & 109.5 \\
\hline $\mathrm{C} 43 \mathrm{a}-\mathrm{C} 44 \mathrm{a}-\mathrm{H} 75 \mathrm{~A}$ & 109.5 \\
\hline $\mathrm{H} 73 \mathrm{a}-\mathrm{C} 44 \mathrm{a}-\mathrm{H} 75 \mathrm{~A}$ & 109.5 \\
\hline $\mathrm{H} 74 \mathrm{a}-\mathrm{C} 44 \mathrm{a}-\mathrm{H} 75 \mathrm{~A}$ & 109.5 \\
\hline $\mathrm{C} 43 \mathrm{~b}-\mathrm{C} 44 \mathrm{~b}-\mathrm{H} 73 \mathrm{~B}$ & 109.5 \\
\hline $\mathrm{C} 43 \mathrm{~b}-\mathrm{C} 44 \mathrm{~b}-\mathrm{H} 74 \mathrm{~B}$ & 109.5 \\
\hline $\mathrm{H} 73 \mathrm{~b}-\mathrm{C} 44 \mathrm{~b}-\mathrm{H} 74 \mathrm{~B}$ & 109.5 \\
\hline $\mathrm{C} 43 \mathrm{~b}-\mathrm{C} 44 \mathrm{~b}-\mathrm{H} 75 \mathrm{~B}$ & 109.5 \\
\hline $\mathrm{H} 73 \mathrm{~b}-\mathrm{C} 44 \mathrm{~b}-\mathrm{H} 75 \mathrm{~B}$ & 109.5 \\
\hline $\mathrm{H} 74 \mathrm{~b}-\mathrm{C} 44 \mathrm{~b}-\mathrm{H} 75 \mathrm{~B}$ & 109.5 \\
\hline $\mathrm{C} 43 \mathrm{a}-\mathrm{C} 45 \mathrm{a}-\mathrm{H} 76 \mathrm{~A}$ & 109.5 \\
\hline $\mathrm{C} 43 \mathrm{a}-\mathrm{C} 45 \mathrm{a}-\mathrm{H} 77 \mathrm{~A}$ & 109.5 \\
\hline $\mathrm{H} 76 \mathrm{a}-\mathrm{C} 45 \mathrm{a}-\mathrm{H} 77 \mathrm{~A}$ & 109.5 \\
\hline $\mathrm{C} 43 \mathrm{a}-\mathrm{C} 45 \mathrm{a}-\mathrm{H} 78 \mathrm{~A}$ & 109.5 \\
\hline $\mathrm{H} 76 \mathrm{a}-\mathrm{C} 45 \mathrm{a}-\mathrm{H} 78 \mathrm{~A}$ & 109.5 \\
\hline $\mathrm{H} 77 \mathrm{a}-\mathrm{C} 45 \mathrm{a}-\mathrm{H} 78 \mathrm{~A}$ & 109.5 \\
\hline $\mathrm{C} 43 \mathrm{~b}-\mathrm{C} 45 \mathrm{~b}-\mathrm{H} 76 \mathrm{~B}$ & 109.5 \\
\hline $\mathrm{C} 43 \mathrm{~b}-\mathrm{C} 45 \mathrm{~b}-\mathrm{H} 77 \mathrm{~B}$ & 109.5 \\
\hline $\mathrm{H} 76 \mathrm{~b}-\mathrm{C} 45 \mathrm{~b}-\mathrm{H} 77 \mathrm{~B}$ & 109.5 \\
\hline $\mathrm{C} 43 \mathrm{~b}-\mathrm{C} 45 \mathrm{~b}-\mathrm{H} 78 \mathrm{~B}$ & 109.5 \\
\hline $\mathrm{H} 76 \mathrm{~b}-\mathrm{C} 45 \mathrm{~b}-\mathrm{H} 78 \mathrm{~B}$ & 109.5 \\
\hline $\mathrm{H} 77 \mathrm{~b}-\mathrm{C} 45 \mathrm{~b}-\mathrm{H} 78 \mathrm{~B}$ & 109.5 \\
\hline $\mathrm{N} 3-\mathrm{B}-\mathrm{N} 5$ & $110.3(2)$ \\
\hline $\mathrm{N} 3-\mathrm{B}-\mathrm{N} 1$ & $110.1(2)$ \\
\hline $\mathrm{N} 5-\mathrm{B}-\mathrm{N} 1$ & $110.3(2)$ \\
\hline $\mathrm{N} 3-\mathrm{B}-\mathrm{H} 1$ & 108.7 \\
\hline $\mathrm{N} 5-\mathrm{B}-\mathrm{H} 1$ & 108.7 \\
\hline $\mathrm{N} 1-\mathrm{B}-\mathrm{H} 1$ & 108.7 \\
\hline $\mathrm{C} 3-\mathrm{N} 1-\mathrm{N} 2$ & $109.2(2)$ \\
\hline $\mathrm{C} 3-\mathrm{N} 1-\mathrm{B}$ & $130.6(2)$ \\
\hline $\mathrm{N} 2-\mathrm{N} 1-\mathrm{B}$ & $120.2(2)$ \\
\hline $\mathrm{C} 1-\mathrm{N} 2-\mathrm{N} 1$ & $106.6(2)$ \\
\hline $\mathrm{C} 1-\mathrm{N} 2-\mathrm{EU}$ & $128.40(18)$ \\
\hline $\mathrm{N} 1-\mathrm{N} 2-\mathrm{EU}$ & $124.87(16)$ \\
\hline
\end{tabular}




\begin{tabular}{|c|c|c|c|}
\hline $\mathrm{H} 41-\mathrm{C} 24-\mathrm{H} 40$ & 109.5 & $\mathrm{C} 12-\mathrm{N} 3-\mathrm{N} 4$ & $109.3(2)$ \\
\hline $\mathrm{C} 21-\mathrm{C} 25-\mathrm{C} 27$ & $111.5(3)$ & $\mathrm{C} 12-\mathrm{N} 3-\mathrm{B}$ & $129.4(2)$ \\
\hline $\mathrm{C} 21-\mathrm{C} 25-\mathrm{C} 26$ & $110.5(3)$ & $\mathrm{N} 4-\mathrm{N} 3-\mathrm{B}$ & $121.3(2)$ \\
\hline $\mathrm{C} 27-\mathrm{C} 25-\mathrm{C} 26$ & $110.5(3)$ & $\mathrm{C} 10-\mathrm{N} 4-\mathrm{N} 3$ & $106.6(2)$ \\
\hline $\mathrm{C} 21-\mathrm{C} 25-\mathrm{H} 42$ & 108.1 & $\mathrm{C} 10-\mathrm{N} 4-\mathrm{EU}$ & $127.79(18)$ \\
\hline $\mathrm{C} 27-\mathrm{C} 25-\mathrm{H} 42$ & 108.1 & $\mathrm{~N} 3-\mathrm{N} 4-\mathrm{EU}$ & $124.67(17)$ \\
\hline $\mathrm{C} 26-\mathrm{C} 25-\mathrm{H} 42$ & 108.1 & $\mathrm{C} 21-\mathrm{N} 5-\mathrm{N} 6$ & $109.7(2)$ \\
\hline $\mathrm{C} 25-\mathrm{C} 26-\mathrm{H} 45$ & 109.5 & $\mathrm{C} 21-\mathrm{N} 5-\mathrm{B}$ & $130.1(2)$ \\
\hline $\mathrm{C} 25-\mathrm{C} 26-\mathrm{H} 44$ & 109.5 & N6-N5-B & $120.1(2)$ \\
\hline $\mathrm{H} 45-\mathrm{C} 26-\mathrm{H} 44$ & 109.5 & $\mathrm{C} 19-\mathrm{N} 6-\mathrm{N} 5$ & $106.6(2)$ \\
\hline $\mathrm{C} 25-\mathrm{C} 26-\mathrm{H} 43$ & 109.5 & C19-N6-EU & $126.86(19)$ \\
\hline $\mathrm{H} 45-\mathrm{C} 26-\mathrm{H} 43$ & 109.5 & N5-N6-EU & $126.29(16)$ \\
\hline $\mathrm{H} 44-\mathrm{C} 26-\mathrm{H} 43$ & 109.5 & $\mathrm{C} 30-\mathrm{N} 7-\mathrm{N} 8$ & $113.1(3)$ \\
\hline $\mathrm{C} 25-\mathrm{C} 27-\mathrm{H} 47$ & 109.5 & $\mathrm{C} 30-\mathrm{N} 7-\mathrm{H} 2$ & 123.5 \\
\hline $\mathrm{C} 25-\mathrm{C} 27-\mathrm{H} 46$ & 109.5 & $\mathrm{~N} 8-\mathrm{N} 7-\mathrm{H} 2$ & 123.5 \\
\hline $\mathrm{H} 47-\mathrm{C} 27-\mathrm{H} 46$ & 109.5 & $\mathrm{C} 28-\mathrm{N} 8-\mathrm{N} 7$ & $104.0(2)$ \\
\hline $\mathrm{C} 25-\mathrm{C} 27-\mathrm{H} 48$ & 109.5 & $\mathrm{C} 28-\mathrm{N} 8-\mathrm{EU}$ & $145.8(2)$ \\
\hline $\mathrm{H} 47-\mathrm{C} 27-\mathrm{H} 48$ & 109.5 & N7-N8-EU & $109.68(17)$ \\
\hline $\mathrm{H} 46-\mathrm{C} 27-\mathrm{H} 48$ & 109.5 & $\mathrm{C} 39-\mathrm{N} 9-\mathrm{N} 10$ & $113.3(2)$ \\
\hline $\mathrm{N} 8-\mathrm{C} 28-\mathrm{C} 29$ & $110.7(3)$ & $\mathrm{C} 39-\mathrm{N} 9-\mathrm{H} 3$ & 123.4 \\
\hline $\mathrm{N} 8-\mathrm{C} 28-\mathrm{C} 31$ & $120.3(3)$ & $\mathrm{N} 10-\mathrm{N} 9-\mathrm{H} 3$ & 123.4 \\
\hline $\mathrm{C} 29-\mathrm{C} 28-\mathrm{C} 31$ & $129.1(3)$ & $\mathrm{C} 37-\mathrm{N} 10-\mathrm{N} 9$ & $104.3(2)$ \\
\hline $\mathrm{C} 30-\mathrm{C} 29-\mathrm{C} 28$ & $106.2(3)$ & $\mathrm{C} 37-\mathrm{N} 10-\mathrm{EU}$ & $142.21(19)$ \\
\hline $\mathrm{C} 30-\mathrm{C} 29-\mathrm{H} 49$ & 126.9 & N9-N10-EU & $113.47(18)$ \\
\hline $\mathrm{C} 28-\mathrm{C} 29-\mathrm{H} 49$ & 126.9 & N6-EU-N4 & $68.39(7)$ \\
\hline $\mathrm{N} 7-\mathrm{C} 30-\mathrm{C} 29$ & $106.0(3)$ & $\mathrm{N} 6-\mathrm{EU}-\mathrm{N} 2$ & $72.16(7)$ \\
\hline $\mathrm{N} 7-\mathrm{C} 30-\mathrm{C} 34 \mathrm{~A}$ & $121.4(3)$ & $\mathrm{N} 4-\mathrm{EU}-\mathrm{N} 2$ & $73.92(7)$ \\
\hline $\mathrm{C} 29-\mathrm{C} 30-\mathrm{C} 34 \mathrm{~A}$ & $132.6(3)$ & N6-EU-N10 & $137.43(7)$ \\
\hline N7-C30-C34B & $121.4(3)$ & $\mathrm{N} 4-\mathrm{EU}-\mathrm{N} 10$ & $76.75(7)$ \\
\hline $\mathrm{C} 29-\mathrm{C} 30-\mathrm{C} 34 \mathrm{~B}$ & $132.6(3)$ & $\mathrm{N} 2-\mathrm{EU}-\mathrm{N} 10$ & $75.37(7)$ \\
\hline $\mathrm{C} 28-\mathrm{C} 31-\mathrm{C} 32$ & $110.7(3)$ & N6-EU-N8 & $75.95(7)$ \\
\hline $\mathrm{C} 28-\mathrm{C} 31-\mathrm{C} 33$ & $111.8(3)$ & $\mathrm{N} 4-\mathrm{EU}-\mathrm{N} 8$ & $138.80(7)$ \\
\hline $\mathrm{C} 32-\mathrm{C} 31-\mathrm{C} 33$ & $110.7(3)$ & $\mathrm{N} 2-\mathrm{EU}-\mathrm{N} 8$ & $76.11(7)$ \\
\hline $\mathrm{C} 28-\mathrm{C} 31-\mathrm{H} 50$ & 107.8 & $\mathrm{~N} 10-\mathrm{EU}-\mathrm{N} 8$ & $121.58(8)$ \\
\hline $\mathrm{C} 32-\mathrm{C} 31-\mathrm{H} 50$ & 107.8 & N6-EU-I & $119.00(5)$ \\
\hline $\mathrm{C} 33-\mathrm{C} 31-\mathrm{H} 50$ & 107.8 & $\mathrm{~N} 4-\mathrm{EU}-\mathrm{I}$ & $117.23(5)$ \\
\hline C31-C32-H53 & 109.5 & $\mathrm{~N} 2-\mathrm{EU}-\mathrm{I}$ & $165.92(5)$ \\
\hline $\mathrm{C} 31-\mathrm{C} 32-\mathrm{H} 51$ & 109.5 & $\mathrm{~N} 10-\mathrm{EU}-\mathrm{I}$ & $98.11(5)$ \\
\hline $\mathrm{H} 53-\mathrm{C} 32-\mathrm{H} 51$ & 109.5 & N8-EU-I & $97.58(5)$ \\
\hline $\mathrm{N} 2-\mathrm{C} 1-\mathrm{C} 2-\mathrm{C} 3$ & $-0.7(3)$ & $\mathrm{N} 9-\mathrm{C} 39-\mathrm{C} 43 \mathrm{~b}-\mathrm{C} 44 \mathrm{~b}$ & $-75.4(13)$ \\
\hline $\mathrm{C} 4-\mathrm{C} 1-\mathrm{C} 2-\mathrm{C} 3$ & $-179.8(3)$ & $\mathrm{C} 38-\mathrm{C} 39-\mathrm{C} 43 \mathrm{~b}-\mathrm{C} 44 \mathrm{~b}$ & $105.1(13)$ \\
\hline $\mathrm{C} 1-\mathrm{C} 2-\mathrm{C} 3-\mathrm{N} 1$ & $0.4(3)$ & $\mathrm{C} 2-\mathrm{C} 3-\mathrm{N} 1-\mathrm{N} 2$ & $0.0(3)$ \\
\hline $\mathrm{C} 1-\mathrm{C} 2-\mathrm{C} 3-\mathrm{C} 7$ & $-177.4(3)$ & $\mathrm{C} 7-\mathrm{C} 3-\mathrm{N} 1-\mathrm{N} 2$ & $177.9(3)$ \\
\hline $\mathrm{N} 2-\mathrm{C} 1-\mathrm{C} 4-\mathrm{C} 6$ & $-111.6(3)$ & $\mathrm{C} 2-\mathrm{C} 3-\mathrm{N} 1-\mathrm{B}$ & $-178.4(3)$ \\
\hline $\mathrm{C} 2-\mathrm{C} 1-\mathrm{C} 4-\mathrm{C} 6$ & $67.4(4)$ & $\mathrm{C} 7-\mathrm{C} 3-\mathrm{N} 1-\mathrm{B}$ & $-0.5(5)$ \\
\hline $\mathrm{N} 2-\mathrm{C} 1-\mathrm{C} 4-\mathrm{C} 5$ & $125.1(3)$ & $\mathrm{N} 3-\mathrm{B}-\mathrm{N} 1-\mathrm{C} 3$ & $116.0(3)$ \\
\hline $\mathrm{C} 2-\mathrm{C} 1-\mathrm{C} 4-\mathrm{C} 5$ & $-55.9(4)$ & $\mathrm{N} 5-\mathrm{B}-\mathrm{N} 1-\mathrm{C} 3$ & $-121.9(3)$ \\
\hline
\end{tabular}




\begin{tabular}{|c|c|}
\hline $\mathrm{N} 1-\mathrm{C} 3-\mathrm{C} 7-\mathrm{C} 9$ & $-92.6(3)$ \\
\hline $\mathrm{C} 2-\mathrm{C} 3-\mathrm{C} 7-\mathrm{C} 9$ & $84.9(4)$ \\
\hline $\mathrm{N} 1-\mathrm{C} 3-\mathrm{C} 7-\mathrm{C} 8$ & $144.6(3)$ \\
\hline $\mathrm{C} 2-\mathrm{C} 3-\mathrm{C} 7-\mathrm{C} 8$ & $-37.9(4)$ \\
\hline $\mathrm{N} 4-\mathrm{C} 10-\mathrm{C} 11-\mathrm{C} 12$ & $-1.7(3)$ \\
\hline $\mathrm{C} 13-\mathrm{C} 10-\mathrm{C} 11-\mathrm{C} 12$ & $175.9(3)$ \\
\hline $\mathrm{C} 10-\mathrm{C} 11-\mathrm{C} 12-\mathrm{N} 3$ & $1.3(3)$ \\
\hline $\mathrm{C} 10-\mathrm{C} 11-\mathrm{C} 12-\mathrm{C} 16$ & $-174.9(3)$ \\
\hline $\mathrm{N} 4-\mathrm{C} 10-\mathrm{C} 13-\mathrm{C} 15$ & $-127.3(3)$ \\
\hline $\mathrm{C} 11-\mathrm{C} 10-\mathrm{C} 13-\mathrm{C} 15$ & $55.3(4)$ \\
\hline $\mathrm{N} 4-\mathrm{C} 10-\mathrm{C} 13-\mathrm{C} 14$ & $109.1(3)$ \\
\hline $\mathrm{C} 11-\mathrm{C} 10-\mathrm{C} 13-\mathrm{C} 14$ & $-68.3(4)$ \\
\hline $\mathrm{N} 3-\mathrm{C} 12-\mathrm{C} 16-\mathrm{C} 18$ & $150.9(3)$ \\
\hline $\mathrm{C} 11-\mathrm{C} 12-\mathrm{C} 16-\mathrm{C} 18$ & $-33.5(4)$ \\
\hline $\mathrm{N} 3-\mathrm{C} 12-\mathrm{C} 16-\mathrm{C} 17$ & $-86.3(4)$ \\
\hline $\mathrm{C} 11-\mathrm{C} 12-\mathrm{C} 16-\mathrm{C} 17$ & $89.4(4)$ \\
\hline $\mathrm{N} 6-\mathrm{C} 19-\mathrm{C} 20-\mathrm{C} 21$ & $0.2(4)$ \\
\hline $\mathrm{C} 22-\mathrm{C} 19-\mathrm{C} 20-\mathrm{C} 21$ & $-177.3(3)$ \\
\hline $\mathrm{C} 19-\mathrm{C} 20-\mathrm{C} 21-\mathrm{N} 5$ & $-0.3(4)$ \\
\hline $\mathrm{C} 19-\mathrm{C} 20-\mathrm{C} 21-\mathrm{C} 25$ & $-177.6(3)$ \\
\hline N6-C19-C22-C24 & $139.4(3)$ \\
\hline $\mathrm{C} 20-\mathrm{C} 19-\mathrm{C} 22-\mathrm{C} 24$ & $-43.4(5)$ \\
\hline $\mathrm{N} 6-\mathrm{C} 19-\mathrm{C} 22-\mathrm{C} 23$ & $-95.2(4)$ \\
\hline $\mathrm{C} 20-\mathrm{C} 19-\mathrm{C} 22-\mathrm{C} 23$ & $82.1(5)$ \\
\hline $\mathrm{N} 5-\mathrm{C} 21-\mathrm{C} 25-\mathrm{C} 27$ & $-75.4(4)$ \\
\hline $\mathrm{C} 20-\mathrm{C} 21-\mathrm{C} 25-\mathrm{C} 27$ & $101.5(4)$ \\
\hline $\mathrm{N} 5-\mathrm{C} 21-\mathrm{C} 25-\mathrm{C} 26$ & $161.3(3)$ \\
\hline $\mathrm{C} 20-\mathrm{C} 21-\mathrm{C} 25-\mathrm{C} 26$ & $-21.8(5)$ \\
\hline $\mathrm{N} 8-\mathrm{C} 28-\mathrm{C} 29-\mathrm{C} 30$ & $-0.3(4)$ \\
\hline $\mathrm{C} 31-\mathrm{C} 28-\mathrm{C} 29-\mathrm{C} 30$ & $179.0(3)$ \\
\hline $\mathrm{C} 28-\mathrm{C} 29-\mathrm{C} 30-\mathrm{N} 7$ & $0.1(4)$ \\
\hline $\mathrm{C} 28-\mathrm{C} 29-\mathrm{C} 30-\mathrm{C} 34 \mathrm{a}$ & $178.3(4)$ \\
\hline $\mathrm{C} 28-\mathrm{C} 29-\mathrm{C} 30-\mathrm{C} 34 \mathrm{~b}$ & $178.3(4)$ \\
\hline $\mathrm{N} 8-\mathrm{C} 28-\mathrm{C} 31-\mathrm{C} 32$ & $66.9(4)$ \\
\hline $\mathrm{C} 29-\mathrm{C} 28-\mathrm{C} 31-\mathrm{C} 32$ & $-112.3(4)$ \\
\hline $\mathrm{N} 8-\mathrm{C} 28-\mathrm{C} 31-\mathrm{C} 33$ & $-169.1(3)$ \\
\hline $\mathrm{C} 29-\mathrm{C} 28-\mathrm{C} 31-\mathrm{C} 33$ & $11.6(5)$ \\
\hline $\mathrm{N} 7-\mathrm{C} 30-\mathrm{C} 34 \mathrm{a}-\mathrm{C} 36 \mathrm{a}$ & $107.3(6)$ \\
\hline $\mathrm{C} 29-\mathrm{C} 30-\mathrm{C} 34 \mathrm{a}-\mathrm{C} 36 \mathrm{a}$ & $-70.6(7)$ \\
\hline $\mathrm{N} 7-\mathrm{C} 30-\mathrm{C} 34 \mathrm{a}-\mathrm{C} 35$ & $-32.9(5)$ \\
\hline $\mathrm{C} 29-\mathrm{C} 30-\mathrm{C} 34 \mathrm{a}-\mathrm{C} 35$ & $149.1(4)$ \\
\hline $\mathrm{N} 7-\mathrm{C} 30-\mathrm{C} 34 \mathrm{~b}-\mathrm{C} 36 \mathrm{~b}$ & $-178.2(7)$ \\
\hline $\mathrm{C} 29-\mathrm{C} 30-\mathrm{C} 34 \mathrm{~b}-\mathrm{C} 36 \mathrm{~b}$ & $3.8(9)$ \\
\hline $\mathrm{N} 7-\mathrm{C} 30-\mathrm{C} 34 \mathrm{~b}-\mathrm{C} 35$ & $-32.9(5)$ \\
\hline $\mathrm{C} 29-\mathrm{C} 30-\mathrm{C} 34 \mathrm{~b}-\mathrm{C} 35$ & $149.1(4)$ \\
\hline $\mathrm{N} 10-\mathrm{C} 37-\mathrm{C} 38-\mathrm{C} 39$ & $0.8(4)$ \\
\hline $\mathrm{C} 40-\mathrm{C} 37-\mathrm{C} 38-\mathrm{C} 39$ & $-178.8(4)$ \\
\hline $\mathrm{C} 37-\mathrm{C} 38-\mathrm{C} 39-\mathrm{N} 9$ & $-0.8(4)$ \\
\hline
\end{tabular}

$\begin{array}{ll}\text { N3-B-N1-N2 } & -62.2(3) \\ \text { N5-B-N1-N2 } & 59.8(3) \\ \text { C2-C1-N2-N1 } & 0.7(3) \\ \text { C4-C1-N2-N1 } & 179.8(3) \\ \text { C2-C1-N2-EU } & 176.38(19) \\ \text { C4-C1-N2-EU } & -4.5(4) \\ \text { C3-N1-N2-C1 } & -0.4(3) \\ \text { B-N1-N2-C1 } & 178.2(2) \\ \text { C3-N1-N2-EU } & -176.29(17) \\ \text { B-N1-N2-EU } & 2.3(3) \\ \text { C11-C12-N3-N4 } & -0.6(3) \\ \text { C16-C12-N3-N4 } & 175.8(2) \\ \text { C11-C12-N3-B } & -178.8(3) \\ \text { C16-C12-N3-B } & -2.4(4) \\ \text { N5-B-N3-C12 } & 125.1(3) \\ \text { N1-B-N3-C12 } & -112.9(3) \\ \text { N5-B-N3-N4 } & -52.9(3) \\ \text { N1-B-N3-N4 } & 69.1(3) \\ \text { C11-C10-N4-N3 } & 1.3(3) \\ \text { C13-C10-N4-N3 } & -176.5(2) \\ \text { C11-C10-N4-EU } & -168.18(19) \\ \text { C13-C10-N4-EU } & 14.0(4) \\ \text { C12-N3-N4-C10 } & -0.5(3) \\ \text { B-N3-N4-C10 } & 177.9(2) \\ \text { C12-N3-N4-EU } & 169.45(17) \\ \text { B-N3-N4-EU } & -12.2(3) \\ \text { C20-C21-N5-N6 } & 0.2(3) \\ \text { C25-C21-N5-N6 } & 177.7(3) \\ \text { C20-C21-N5-B } & 177.7(3) \\ \text { C25-C21-N5-B } & -4.8(5) \\ \text { N3-B-N5-C21 } & -121.5(3) \\ \text { N1-B-N5-C21 } & 116.6(3) \\ \text { N3-B-N5-N6 } & 55.8(3) \\ \text { N1-B-N5-N6 } & -66.1(3) \\ \text { C20-C19-N6-N5 } & 0.0(3) \\ \text { C22-C19-N6-N5 } & 177.7(3) \\ \text { C20-C19-N6-EU } & 174.9(2) \\ \text { C22-C19-N6-EU } & -7.3(4) \\ \text { C21-N5-N6-C19 } & -0.1(3) \\ \text { B-N5-N6-C19 } & -177.9(2) \\ \text { C21-N5-N6-EU } & -175.07(18) \\ \text { B-N5-N6-EU } & 7.1(3) \\ \text { C29-C30-N7-N8 } & 0.1(4) \\ \text { C34a-C30-N7-N8 } & -178.3(3) \\ \text { C34b-C30-N7-N8 } & -178.3(3) \\ \text { C29-C28-N8-N7 } & 0.4(3) \\ \text { C31-C28-N8-N7 } & -179.0(3) \\ \text { C29-C28-N8-EU } & \\ & \end{array}$




$\begin{array}{ll}\mathrm{C} 37-\mathrm{C} 38-\mathrm{C} 39-\mathrm{C} 43 \mathrm{a} & 178.8(4) \\ \mathrm{C} 37-\mathrm{C} 38-\mathrm{C} 39-\mathrm{C} 43 \mathrm{~b} & 178.8(4) \\ \mathrm{N} 10-\mathrm{C} 37-\mathrm{C} 40-\mathrm{C} 41 & 117.8(4) \\ \mathrm{C} 38-\mathrm{C} 37-\mathrm{C} 40-\mathrm{C} 41 & -62.6(5) \\ \mathrm{N} 10-\mathrm{C} 37-\mathrm{C} 40-\mathrm{C} 42 & -119.1(4) \\ \mathrm{C} 38-\mathrm{C} 37-\mathrm{C} 40-\mathrm{C} 42 & 60.5(5) \\ \mathrm{N} 9-\mathrm{C} 39-\mathrm{C} 43 \mathrm{a}-\mathrm{C} 44 \mathrm{a} & -111.9(7) \\ \mathrm{C} 38-\mathrm{C} 39-\mathrm{C} 43 \mathrm{a}-\mathrm{C} 44 \mathrm{a} & 68.5(8) \\ \mathrm{N} 9-\mathrm{C} 39-\mathrm{C} 43 \mathrm{a}-\mathrm{C} 45 \mathrm{a} & 24.2(7) \\ \mathrm{C} 38-\mathrm{C} 39-\mathrm{C} 43 \mathrm{a}-\mathrm{C} 45 \mathrm{a} & -155.3(6) \\ \mathrm{N} 9-\mathrm{C} 39-\mathrm{C} 43 \mathrm{~b}-\mathrm{C} 45 \mathrm{~b} & 60.8(14) \\ \mathrm{C} 38-\mathrm{C} 39-\mathrm{C} 43 \mathrm{~b}-\mathrm{C} 45 \mathrm{~b} & -118.7(13)\end{array}$

$$
\begin{aligned}
& \mathrm{C} 31-\mathrm{C} 28-\mathrm{N} 8-\mathrm{EU} \\
& \mathrm{C} 30-\mathrm{N} 7-\mathrm{N} 8-\mathrm{C} 28 \\
& \mathrm{C} 30-\mathrm{N} 7-\mathrm{N} 8-\mathrm{EU} \\
& \mathrm{C} 38-\mathrm{C} 39-\mathrm{N} 9-\mathrm{N} 10 \\
& \mathrm{C} 43 \mathrm{a}-\mathrm{C} 39-\mathrm{N} 9-\mathrm{N} 10 \\
& \mathrm{C} 43 \mathrm{~b}-\mathrm{C} 39-\mathrm{N} 9-\mathrm{N} 10 \\
& \mathrm{C} 38-\mathrm{C} 37-\mathrm{N} 10-\mathrm{N} 9 \\
& \mathrm{C} 40-\mathrm{C} 37-\mathrm{N} 10-\mathrm{N} 9 \\
& \mathrm{C} 38-\mathrm{C} 37-\mathrm{N} 10-\mathrm{EU} \\
& \mathrm{C} 40-\mathrm{C} 37-\mathrm{N} 10-\mathrm{EU} \\
& \mathrm{C} 39-\mathrm{N} 9-\mathrm{N} 10-\mathrm{C} 37 \\
& \mathrm{C} 39-\mathrm{N} 9-\mathrm{N} 10-\mathrm{EU}
\end{aligned}
$$$$
174.1(2)
$$$$
0.6(4)
$$$$
-179.1(3)
$$$$
-179.1(3)
$$$$
-0.5(4)
$$

trans-4,8-Bis(3,5-diisopropylpyrazol-1-yl)-1,3,5,7-tetraisopropylpyrazabole (2)

\section{Crystal data}

$\mathrm{C}_{36} \mathrm{H}_{62} \mathrm{~B}_{2} \mathrm{~N}_{8}$

$M_{r}=628.55$

Monoclinic, $C 2 / \mathrm{c}$

$a=25.7646(11) \AA$

$b=11.2134(3) \AA$

$c=15.0968(7) \AA$

$\beta=118.792(3)^{\circ}$

$V=3822.4(3) \AA^{3}$

$Z=4$

\section{Data collection}

Stoe IPDS 2T diffractometer

Radiation source: fine-focus sealed tube Detector resolution: 6.67 pixels $\mathrm{mm}^{-1}$ area detector scans 10509 measured reflections 3367 independent reflections

\section{Refinement}

Refinement on $F^{2}$

Least-squares matrix: full

$R\left[F^{2}>2 \sigma\left(F^{2}\right)\right]=0.044$

$w R\left(F^{2}\right)=0.104$

$S=1.02$

3367 reflections

238 parameters

0 restraints

Primary atom site location: structure-invariant direct methods

Secondary atom site location: difference Fourier map
$F(000)=1376$

$D_{\mathrm{x}}=1.092 \mathrm{Mg} \mathrm{m}^{-3}$

Mo $K \alpha$ radiation, $\lambda=0.71073 \AA$

Cell parameters from 11890 reflections

$\theta=2.0-26.2^{\circ}$

$\mu=0.07 \mathrm{~mm}^{-1}$

$T=153 \mathrm{~K}$

Plate, colorless

$0.33 \times 0.29 \times 0.13 \mathrm{~mm}$

2594 reflections with $I>2 \sigma(I)$

$R_{\text {int }}=0.046$

$\theta_{\max }=25.0^{\circ}, \theta_{\min }=2.0^{\circ}$

$h=-30 \rightarrow 29$

$k=-12 \rightarrow 13$

$l=-17 \rightarrow 17$

Hydrogen site location: inferred from neighbouring sites

$\mathrm{H}$-atom parameters constrained

$w=1 /\left[\sigma^{2}\left(F_{0}{ }^{2}\right)+(0.050 P)^{2}+1.3932 P\right]$

where $P=\left(F_{\mathrm{o}}^{2}+2 F_{\mathrm{c}}^{2}\right) / 3$

$(\Delta / \sigma)_{\max }<0.001$

$\Delta \rho_{\max }=0.25 \mathrm{e} \AA^{-3}$

$\Delta \rho_{\text {min }}=-0.20 \mathrm{e}^{-3}$

Extinction correction: SHELXL2016

(Sheldrick, 2015),

$\mathrm{Fc}^{*}=\mathrm{kFc}\left[1+0.001 \mathrm{xFc}^{2} \lambda^{3} / \sin (2 \theta)\right]^{-1 / 4}$

Extinction coefficient: 0.0022 (4) 


\section{Special details}

Geometry. All esds (except the esd in the dihedral angle between two 1.s. planes) are estimated using the full covariance matrix. The cell esds are taken into account individually in the estimation of esds in distances, angles and torsion angles; correlations between esds in cell parameters are only used when they are defined by crystal symmetry. An approximate (isotropic) treatment of cell esds is used for estimating esds involving l.s. planes.

Fractional atomic coordinates and isotropic or equivalent isotropic displacement parameters $\left(\AA^{2}\right)$

\begin{tabular}{|c|c|c|c|c|c|}
\hline & $x$ & $y$ & $z$ & $U_{\text {iso }} * / U_{\text {eq }}$ & Occ. $(<1)$ \\
\hline $\mathrm{C} 1$ & $0.32091(7)$ & $0.43231(14)$ & $0.12232(11)$ & $0.0228(3)$ & \\
\hline $\mathrm{C} 2$ & $0.31092(7)$ & $0.42821(15)$ & $0.20414(12)$ & $0.0275(4)$ & \\
\hline $\mathrm{H} 2$ & 0.328176 & 0.478383 & 0.261967 & $0.033^{*}$ & \\
\hline $\mathrm{C} 3$ & $0.27098(7)$ & $0.33708(14)$ & $0.18577(11)$ & $0.0239(3)$ & \\
\hline $\mathrm{C} 4$ & $0.36140(7)$ & $0.51263(14)$ & $0.10426(12)$ & $0.0255(4)$ & \\
\hline H3 & 0.350866 & 0.506956 & 0.031400 & $0.031^{*}$ & \\
\hline $\mathrm{C} 5$ & $0.42566(7)$ & 0.47407 (17) & $0.16846(13)$ & $0.0361(4)$ & \\
\hline H5 & 0.451450 & 0.527573 & 0.155810 & $0.054^{*}$ & \\
\hline H6 & 0.430455 & 0.392253 & 0.150739 & $0.054^{*}$ & \\
\hline $\mathrm{H} 4$ & 0.436389 & 0.477573 & 0.240128 & $0.054 *$ & \\
\hline C6 & $0.35282(9)$ & $0.64149(16)$ & $0.12723(15)$ & $0.0390(4)$ & \\
\hline H9 & 0.378721 & 0.693891 & 0.113901 & $0.059^{*}$ & \\
\hline H8 & 0.362781 & 0.648366 & 0.198397 & $0.059^{*}$ & \\
\hline H7 & 0.311453 & 0.664942 & 0.084152 & $0.059 *$ & \\
\hline $\mathrm{C} 7$ & $0.24394(7)$ & $0.29648(16)$ & $0.24900(12)$ & $0.0302(4)$ & \\
\hline H10 & 0.223957 & 0.218098 & 0.222317 & $0.036^{*}$ & \\
\hline $\mathrm{C} 8$ & $0.29206(10)$ & $0.2793(2)$ & $0.35783(14)$ & $0.0543(6)$ & \\
\hline H11 & 0.274068 & 0.253611 & 0.398945 & $0.081^{*}$ & \\
\hline H12 & 0.313065 & 0.354794 & 0.384237 & $0.081^{*}$ & \\
\hline H13 & 0.320074 & 0.218456 & 0.360467 & $0.081^{*}$ & \\
\hline $\mathrm{C} 9$ & $0.19746(9)$ & $0.38648(18)$ & $0.24156(16)$ & $0.0451(5)$ & \\
\hline H15 & 0.179127 & 0.358161 & 0.281449 & $0.068^{*}$ & \\
\hline H14 & 0.167006 & 0.395218 & 0.170735 & $0.068^{*}$ & \\
\hline H16 & 0.216435 & 0.463780 & 0.267740 & $0.068^{*}$ & \\
\hline $\mathrm{C} 10$ & $0.06813(7)$ & $0.23099(14)$ & $0.02850(12)$ & $0.0257(4)$ & \\
\hline C11 & $0.06359(7)$ & $0.32593(15)$ & $-0.03555(13)$ & $0.0290(4)$ & \\
\hline H17 & 0.031464 & 0.379970 & -0.068315 & $0.035^{*}$ & \\
\hline $\mathrm{C} 12$ & $0.11522(7)$ & $0.32426(14)$ & $-0.04087(11)$ & $0.0242(3)$ & \\
\hline $\mathrm{C} 13$ & $0.02374(7)$ & $0.19290(17)$ & $0.06011(13)$ & $0.0322(4)$ & \\
\hline H18 & 0.039684 & 0.120355 & 0.103467 & $0.039 *$ & \\
\hline $\mathrm{C} 14$ & $0.01516(10)$ & $0.2889(2)$ & $0.12299(18)$ & $0.0527(6)$ & \\
\hline $\mathrm{H} 20$ & -0.011435 & 0.259168 & 0.147452 & $0.079 *$ & \\
\hline H19 & -0.002167 & 0.359966 & 0.081317 & $0.079^{*}$ & \\
\hline $\mathrm{H} 21$ & 0.053547 & 0.309303 & 0.180752 & $0.079 *$ & \\
\hline $\mathrm{C} 15$ & $-0.03477(8)$ & $0.1591(2)$ & $-0.03071(16)$ & $0.0520(6)$ & \\
\hline $\mathrm{H} 24$ & -0.061559 & 0.126983 & -0.007583 & $0.078^{*}$ & \\
\hline $\mathrm{H} 23$ & -0.027873 & 0.098683 & -0.070761 & $0.078^{*}$ & \\
\hline $\mathrm{H} 22$ & -0.052662 & 0.229990 & -0.072424 & $0.078^{*}$ & \\
\hline C16A & $0.13485(7)$ & $0.40591(15)$ & $-0.09797(13)$ & $0.0292(4)$ & $0.649(9)$ \\
\hline
\end{tabular}




\begin{tabular}{|c|c|c|c|c|c|}
\hline $\mathrm{H} 25 \mathrm{~A}$ & 0.168807 & 0.367438 & -0.101562 & $0.035^{*}$ & $0.649(9)$ \\
\hline C17A & $0.1569(3)$ & $0.5251(3)$ & $-0.0392(4)$ & $0.0435(11)$ & $0.649(9)$ \\
\hline $\mathrm{H} 26 \mathrm{~A}$ & 0.171675 & 0.577049 & -0.074376 & $0.065^{*}$ & $0.649(9)$ \\
\hline $\mathrm{H} 27 \mathrm{~A}$ & 0.188876 & 0.508864 & 0.029262 & $0.065^{*}$ & $0.649(9)$ \\
\hline $\mathrm{H} 28 \mathrm{~A}$ & 0.124079 & 0.564576 & -0.035502 & $0.065 *$ & $0.649(9)$ \\
\hline C18A & $0.0865(2)$ & $0.4274(6)$ & $-0.2035(3)$ & $0.0535(14)$ & $0.649(9)$ \\
\hline $\mathrm{H} 29 \mathrm{~A}$ & 0.101968 & 0.474541 & -0.240313 & $0.080 *$ & $0.649(9)$ \\
\hline $\mathrm{H} 30 \mathrm{~A}$ & 0.054076 & 0.470950 & -0.201581 & $0.080 *$ & $0.649(9)$ \\
\hline H31A & 0.071760 & 0.350805 & -0.237778 & $0.080^{*}$ & $0.649(9)$ \\
\hline C16B & $0.13485(7)$ & $0.40591(15)$ & -0.09797 (13) & $0.0292(4)$ & $0.351(9)$ \\
\hline $\mathrm{H} 25 \mathrm{~B}$ & 0.178789 & 0.399759 & -0.068850 & $0.035^{*}$ & $0.351(9)$ \\
\hline C17B & $0.1037(4)$ & $0.3646(8)$ & $-0.2133(5)$ & $0.045(2)$ & $0.351(9)$ \\
\hline H26B & 0.115240 & 0.418278 & -0.251917 & $0.067 *$ & $0.351(9)$ \\
\hline H27B & 0.060562 & 0.366928 & -0.241231 & $0.067^{*}$ & $0.351(9)$ \\
\hline H28B & 0.116013 & 0.283030 & -0.217459 & $0.067 *$ & $0.351(9)$ \\
\hline C18B & $0.1187(6)$ & $0.5326(6)$ & $-0.0920(9)$ & $0.053(3)$ & $0.351(9)$ \\
\hline H29B & 0.132204 & 0.583712 & -0.129447 & $0.079^{*}$ & $0.351(9)$ \\
\hline H30B & 0.137625 & 0.557807 & -0.021063 & $0.079 *$ & $0.351(9)$ \\
\hline H31B & 0.075580 & 0.539310 & -0.121428 & $0.079 *$ & $0.351(9)$ \\
\hline B & $0.21039(7)$ & $0.18853(16)$ & $0.04185(12)$ & $0.0209(4)$ & \\
\hline $\mathrm{H} 1$ & 0.220947 & 0.117181 & 0.086985 & $0.025^{*}$ & \\
\hline N1 & $0.25728(5)$ & $0.28776(11)$ & 0.09607 (9) & $0.0205(3)$ & \\
\hline N2 & $0.28824(5)$ & $0.34639(11)$ & $0.05681(9)$ & $0.0198(3)$ & \\
\hline N3 & $0.14859(5)$ & $0.23196(11)$ & $0.01778(9)$ & $0.0207(3)$ & \\
\hline N4 & $0.11934(6)$ & $0.17386(12)$ & $0.06116(9)$ & 0.0235 & \\
\hline
\end{tabular}

Atomic displacement parameters $\left(\AA^{2}\right)$

\begin{tabular}{lllllll}
\hline & $U^{11}$ & $U^{22}$ & $U^{33}$ & $U^{12}$ & $U^{13}$ & $U^{23}$ \\
\hline C1 & $0.0190(8)$ & $0.0221(8)$ & $0.0248(8)$ & $-0.0002(6)$ & $0.0085(6)$ & $-0.0021(6)$ \\
C2 & $0.0270(9)$ & $0.0295(9)$ & $0.0254(8)$ & $-0.0041(7)$ & $0.0121(7)$ & $-0.0077(7)$ \\
C3 & $0.0220(8)$ & $0.0275(8)$ & $0.0210(7)$ & $-0.0009(7)$ & $0.0095(6)$ & $-0.0027(6)$ \\
C4 & $0.0244(8)$ & $0.0246(8)$ & $0.0256(8)$ & $-0.0043(7)$ & $0.0105(7)$ & $-0.0019(7)$ \\
C5 & $0.0250(9)$ & $0.0431(10)$ & $0.0374(10)$ & $-0.0067(8)$ & $0.0128(8)$ & $-0.0010(8)$ \\
C6 & $0.0451(11)$ & $0.0273(9)$ & $0.0485(11)$ & $-0.0060(8)$ & $0.0255(10)$ & $-0.0046(8)$ \\
C7 & $0.0306(9)$ & $0.0372(10)$ & $0.0262(8)$ & $-0.0092(8)$ & $0.0165(7)$ & $-0.0063(7)$ \\
C8 & $0.0474(12)$ & $0.0863(17)$ & $0.0293(10)$ & $-0.0183(12)$ & $0.0185(9)$ & $0.0022(10)$ \\
C9 & $0.0496(12)$ & $0.0469(12)$ & $0.0550(12)$ & $-0.0104(10)$ & $0.0382(10)$ & $-0.0136(10)$ \\
C10 & $0.0213(8)$ & $0.0295(9)$ & $0.0277(8)$ & $-0.0015(7)$ & $0.0130(7)$ & $-0.0006(7)$ \\
C11 & $0.0234(8)$ & $0.0294(9)$ & $0.0350(9)$ & $0.0046(7)$ & $0.0147(7)$ & $0.0040(7)$ \\
C12 & $0.0234(8)$ & $0.0229(8)$ & $0.0265(8)$ & $0.0023(7)$ & $0.0122(7)$ & $0.0009(6)$ \\
C13 & $0.0262(9)$ & $0.0385(10)$ & $0.0368(9)$ & $-0.0005(8)$ & $0.0190(8)$ & $0.0045(8)$ \\
C14 & $0.0529(13)$ & $0.0612(14)$ & $0.0649(14)$ & $-0.0020(11)$ & $0.0450(12)$ & $-0.0062(11)$ \\
C15 & $0.0312(10)$ & $0.0748(15)$ & $0.0488(12)$ & $-0.0145(10)$ & $0.0184(9)$ & $0.0055(11)$ \\
C16A & $0.0271(9)$ & $0.0287(9)$ & $0.0360(9)$ & $0.0053(7)$ & $0.0183(8)$ & $0.0086(7)$ \\
C17A & $0.054(3)$ & $0.0287(17)$ & $0.056(2)$ & $-0.0048(18)$ & $0.033(2)$ & $0.0051(16)$ \\
C18A & $0.045(2)$ & $0.067(3)$ & $0.0401(19)$ & $-0.010(2)$ & $0.0142(17)$ & $0.020(2)$ \\
C16B & $0.0271(9)$ & $0.0287(9)$ & $0.0360(9)$ & $0.0053(7)$ & $0.0183(8)$ & $0.0086(7)$
\end{tabular}




\begin{tabular}{lllllll} 
C17B & $0.048(4)$ & $0.053(5)$ & $0.036(3)$ & $0.001(3)$ & $0.022(3)$ & $0.012(3)$ \\
C18B & $0.079(7)$ & $0.030(3)$ & $0.075(6)$ & $0.009(4)$ & $0.058(6)$ & $0.013(3)$ \\
B & $0.0200(9)$ & $0.0204(9)$ & $0.0215(8)$ & $-0.0001(7)$ & $0.0093(7)$ & $0.0012(7)$ \\
N1 & $0.0186(6)$ & $0.0228(7)$ & $0.0205(6)$ & $-0.0007(5)$ & $0.0098(5)$ & $-0.0005(5)$ \\
N2 & $0.0167(6)$ & $0.0214(7)$ & $0.0215(6)$ & $-0.0011(5)$ & $0.0095(5)$ & $0.0001(5)$ \\
N3 & $0.0199(7)$ & $0.0215(7)$ & $0.0226(6)$ & $-0.0008(5)$ & $0.0117(5)$ & $0.0009(5)$ \\
N4 & $0.0224(7)$ & $0.0261(7)$ & $0.0250(7)$ & $-0.0028(6)$ & $0.0138(6)$ & $-0.0002(5)$ \\
\hline
\end{tabular}

Geometric parameters $\left(A,{ }^{\circ}\right)$

\begin{tabular}{|c|c|c|c|}
\hline $\mathrm{C} 1-\mathrm{N} 2$ & $1.3464(19)$ & $\mathrm{C} 13-\mathrm{C} 14$ & $1.520(3)$ \\
\hline $\mathrm{C} 1-\mathrm{C} 2$ & $1.379(2)$ & $\mathrm{C} 13-\mathrm{H} 18$ & 1.0000 \\
\hline $\mathrm{C} 1-\mathrm{C} 4$ & $1.499(2)$ & $\mathrm{C} 14-\mathrm{H} 20$ & 0.9800 \\
\hline $\mathrm{C} 2-\mathrm{C} 3$ & $1.380(2)$ & C14-H19 & 0.9800 \\
\hline $\mathrm{C} 2-\mathrm{H} 2$ & 0.9500 & $\mathrm{C} 14-\mathrm{H} 21$ & 0.9800 \\
\hline $\mathrm{C} 3-\mathrm{N} 1$ & $1.3431(19)$ & $\mathrm{C} 15-\mathrm{H} 24$ & 0.9800 \\
\hline $\mathrm{C} 3-\mathrm{C} 7$ & $1.498(2)$ & $\mathrm{C} 15-\mathrm{H} 23$ & 0.9800 \\
\hline $\mathrm{C} 4-\mathrm{C} 5$ & $1.524(2)$ & $\mathrm{C} 15-\mathrm{H} 22$ & 0.9800 \\
\hline $\mathrm{C} 4-\mathrm{C} 6$ & $1.527(2)$ & $\mathrm{C} 16 \mathrm{a}-\mathrm{C} 18 \mathrm{~A}$ & $1.496(4)$ \\
\hline $\mathrm{C} 4-\mathrm{H} 3$ & 1.0000 & $\mathrm{C} 16 \mathrm{a}-\mathrm{C} 17 \mathrm{~A}$ & $1.552(4)$ \\
\hline $\mathrm{C} 5-\mathrm{H} 5$ & 0.9800 & $\mathrm{C} 16 \mathrm{a}-\mathrm{H} 25 \mathrm{~A}$ & 1.0000 \\
\hline $\mathrm{C} 5-\mathrm{H} 6$ & 0.9800 & $\mathrm{C} 17 \mathrm{a}-\mathrm{H} 26 \mathrm{~A}$ & 0.9800 \\
\hline $\mathrm{C} 5-\mathrm{H} 4$ & 0.9800 & $\mathrm{C} 17 \mathrm{a}-\mathrm{H} 27 \mathrm{~A}$ & 0.9800 \\
\hline $\mathrm{C} 6-\mathrm{H} 9$ & 0.9800 & $\mathrm{C} 17 \mathrm{a}-\mathrm{H} 28 \mathrm{~A}$ & 0.9800 \\
\hline $\mathrm{C} 6-\mathrm{H} 8$ & 0.9800 & $\mathrm{C} 18 \mathrm{a}-\mathrm{H} 29 \mathrm{~A}$ & 0.9800 \\
\hline $\mathrm{C} 6-\mathrm{H} 7$ & 0.9800 & $\mathrm{C} 18 \mathrm{a}-\mathrm{H} 30 \mathrm{~A}$ & 0.9800 \\
\hline $\mathrm{C} 7-\mathrm{C} 8$ & $1.520(3)$ & $\mathrm{C} 18 \mathrm{a}-\mathrm{H} 31 \mathrm{~A}$ & 0.9800 \\
\hline $\mathrm{C} 7-\mathrm{C} 9$ & $1.528(3)$ & $\mathrm{C} 16 \mathrm{~b}-\mathrm{C} 18 \mathrm{~B}$ & $1.496(7)$ \\
\hline $\mathrm{C} 7-\mathrm{H} 10$ & 1.0000 & $\mathrm{C} 16 \mathrm{~b}-\mathrm{C} 17 \mathrm{~B}$ & $1.595(7)$ \\
\hline $\mathrm{C} 8-\mathrm{H} 11$ & 0.9800 & $\mathrm{C} 16 \mathrm{~b}-\mathrm{H} 25 \mathrm{~B}$ & 1.0000 \\
\hline $\mathrm{C} 8-\mathrm{H} 12$ & 0.9800 & $\mathrm{C} 17 \mathrm{~b}-\mathrm{H} 26 \mathrm{~B}$ & 0.9800 \\
\hline $\mathrm{C} 8-\mathrm{H} 13$ & 0.9800 & $\mathrm{C} 17 \mathrm{~b}-\mathrm{H} 27 \mathrm{~B}$ & 0.9800 \\
\hline $\mathrm{C} 9-\mathrm{H} 15$ & 0.9800 & $\mathrm{C} 17 \mathrm{~b}-\mathrm{H} 28 \mathrm{~B}$ & 0.9800 \\
\hline $\mathrm{C} 9-\mathrm{H} 14$ & 0.9800 & $\mathrm{C} 18 \mathrm{~b}-\mathrm{H} 29 \mathrm{~B}$ & 0.9800 \\
\hline C9-H16 & 0.9800 & $\mathrm{C} 18 \mathrm{~b}-\mathrm{H} 30 \mathrm{~B}$ & 0.9800 \\
\hline $\mathrm{C} 10-\mathrm{N} 4$ & $1.330(2)$ & $\mathrm{C} 18 \mathrm{~b}-\mathrm{H} 31 \mathrm{~B}$ & 0.9800 \\
\hline $\mathrm{C} 10-\mathrm{C} 11$ & $1.405(2)$ & $\mathrm{B}-\mathrm{N} 3$ & $1.532(2)$ \\
\hline $\mathrm{C} 10-\mathrm{C} 13$ & $1.498(2)$ & $\mathrm{B}-\mathrm{N} 1$ & $1.554(2)$ \\
\hline $\mathrm{C} 11-\mathrm{C} 12$ & $1.371(2)$ & $\mathrm{B}-\mathrm{N} 2^{\mathrm{i}}$ & $1.557(2)$ \\
\hline $\mathrm{C} 11-\mathrm{H} 17$ & 0.9500 & $\mathrm{~B}-\mathrm{H} 1$ & 1.0000 \\
\hline $\mathrm{C} 12-\mathrm{N} 3$ & $1.364(2)$ & $\mathrm{N} 1-\mathrm{N} 2$ & $1.3693(16)$ \\
\hline $\mathrm{C} 12-\mathrm{C} 16 \mathrm{~A}$ & $1.502(2)$ & $\mathrm{N} 2-\mathrm{B}^{\mathrm{i}}$ & $1.557(2)$ \\
\hline $\mathrm{C} 12-\mathrm{C} 16 \mathrm{~B}$ & $1.502(2)$ & $\mathrm{N} 3-\mathrm{N} 4$ & $1.3772(17)$ \\
\hline $\mathrm{C} 13-\mathrm{C} 15$ & $1.518(3)$ & & \\
\hline $\mathrm{N} 2-\mathrm{C} 1-\mathrm{C} 2$ & $108.29(13)$ & $\mathrm{C} 13-\mathrm{C} 14-\mathrm{H} 19$ & 109.5 \\
\hline $\mathrm{N} 2-\mathrm{C} 1-\mathrm{C} 4$ & $122.67(13)$ & $\mathrm{H} 20-\mathrm{C} 14-\mathrm{H} 19$ & 109.5 \\
\hline $\mathrm{C} 2-\mathrm{C} 1-\mathrm{C} 4$ & $129.02(14)$ & $\mathrm{C} 13-\mathrm{C} 14-\mathrm{H} 21$ & 109.5 \\
\hline
\end{tabular}




\begin{tabular}{|c|c|}
\hline $\mathrm{C} 1-\mathrm{C} 2-\mathrm{C} 3$ & $106.71(14)$ \\
\hline $\mathrm{C} 1-\mathrm{C} 2-\mathrm{H} 2$ & 126.6 \\
\hline $\mathrm{C} 3-\mathrm{C} 2-\mathrm{H} 2$ & 126.6 \\
\hline $\mathrm{N} 1-\mathrm{C} 3-\mathrm{C} 2$ & $108.41(13)$ \\
\hline $\mathrm{N} 1-\mathrm{C} 3-\mathrm{C} 7$ & $122.71(14)$ \\
\hline $\mathrm{C} 2-\mathrm{C} 3-\mathrm{C} 7$ & $128.86(14)$ \\
\hline $\mathrm{C} 1-\mathrm{C} 4-\mathrm{C} 5$ & $110.60(13)$ \\
\hline $\mathrm{C} 1-\mathrm{C} 4-\mathrm{C} 6$ & $109.73(13)$ \\
\hline $\mathrm{C} 5-\mathrm{C} 4-\mathrm{C} 6$ & $110.85(14)$ \\
\hline $\mathrm{C} 1-\mathrm{C} 4-\mathrm{H} 3$ & 108.5 \\
\hline $\mathrm{C} 5-\mathrm{C} 4-\mathrm{H} 3$ & 108.5 \\
\hline $\mathrm{C} 6-\mathrm{C} 4-\mathrm{H} 3$ & 108.5 \\
\hline $\mathrm{C} 4-\mathrm{C} 5-\mathrm{H} 5$ & 109.5 \\
\hline $\mathrm{C} 4-\mathrm{C} 5-\mathrm{H} 6$ & 109.5 \\
\hline $\mathrm{H} 5-\mathrm{C} 5-\mathrm{H} 6$ & 109.5 \\
\hline $\mathrm{C} 4-\mathrm{C} 5-\mathrm{H} 4$ & 109.5 \\
\hline $\mathrm{H} 5-\mathrm{C} 5-\mathrm{H} 4$ & 109.5 \\
\hline $\mathrm{H} 6-\mathrm{C} 5-\mathrm{H} 4$ & 109.5 \\
\hline $\mathrm{C} 4-\mathrm{C} 6-\mathrm{H} 9$ & 109.5 \\
\hline $\mathrm{C} 4-\mathrm{C} 6-\mathrm{H} 8$ & 109.5 \\
\hline $\mathrm{H} 9-\mathrm{C} 6-\mathrm{H} 8$ & 109.5 \\
\hline $\mathrm{C} 4-\mathrm{C} 6-\mathrm{H} 7$ & 109.5 \\
\hline $\mathrm{H} 9-\mathrm{C} 6-\mathrm{H} 7$ & 109.5 \\
\hline $\mathrm{H} 8-\mathrm{C} 6-\mathrm{H} 7$ & 109.5 \\
\hline $\mathrm{C} 3-\mathrm{C} 7-\mathrm{C} 8$ & $109.85(14)$ \\
\hline $\mathrm{C} 3-\mathrm{C} 7-\mathrm{C} 9$ & $109.93(14)$ \\
\hline $\mathrm{C} 8-\mathrm{C} 7-\mathrm{C} 9$ & $111.42(16)$ \\
\hline $\mathrm{C} 3-\mathrm{C} 7-\mathrm{H} 10$ & 108.5 \\
\hline $\mathrm{C} 8-\mathrm{C} 7-\mathrm{H} 10$ & 108.5 \\
\hline $\mathrm{C} 9-\mathrm{C} 7-\mathrm{H} 10$ & 108.5 \\
\hline $\mathrm{C} 7-\mathrm{C} 8-\mathrm{H} 11$ & 109.5 \\
\hline $\mathrm{C} 7-\mathrm{C} 8-\mathrm{H} 12$ & 109.5 \\
\hline $\mathrm{H} 11-\mathrm{C} 8-\mathrm{H} 12$ & 109.5 \\
\hline $\mathrm{C} 7-\mathrm{C} 8-\mathrm{H} 13$ & 109.5 \\
\hline $\mathrm{H} 11-\mathrm{C} 8-\mathrm{H} 13$ & 109.5 \\
\hline $\mathrm{H} 12-\mathrm{C} 8-\mathrm{H} 13$ & 109.5 \\
\hline $\mathrm{C} 7-\mathrm{C} 9-\mathrm{H} 15$ & 109.5 \\
\hline $\mathrm{C} 7-\mathrm{C} 9-\mathrm{H} 14$ & 109.5 \\
\hline $\mathrm{H} 15-\mathrm{C} 9-\mathrm{H} 14$ & 109.5 \\
\hline $\mathrm{C} 7-\mathrm{C} 9-\mathrm{H} 16$ & 109.5 \\
\hline $\mathrm{H} 15-\mathrm{C} 9-\mathrm{H} 16$ & 109.5 \\
\hline $\mathrm{H} 14-\mathrm{C} 9-\mathrm{H} 16$ & 109.5 \\
\hline $\mathrm{N} 4-\mathrm{C} 10-\mathrm{C} 11$ & $111.05(13)$ \\
\hline $\mathrm{N} 4-\mathrm{C} 10-\mathrm{C} 13$ & $121.20(14)$ \\
\hline $\mathrm{C} 11-\mathrm{C} 10-\mathrm{C} 13$ & $127.74(15)$ \\
\hline $\mathrm{C} 12-\mathrm{C} 11-\mathrm{C} 10$ & $105.58(14)$ \\
\hline $\mathrm{C} 12-\mathrm{C} 11-\mathrm{H} 17$ & 127.2 \\
\hline $\mathrm{C} 10-\mathrm{C} 11-\mathrm{H} 17$ & 127.2 \\
\hline
\end{tabular}

109.5

109.5

109.5

109.5

109.5

109.5

109.5

109.5

$111.80(19)$

$111.0(3)$

109.18 (17)

108.3

108.3

108.3

109.5

109.5

109.5

109.5

109.5

109.5

109.5

109.5

109.5

109.5

109.5

109.5

$111.3(3)$

$109.5(5)$

108.5 (3)

109.2

109.2

109.2

109.5

109.5

109.5

109.5

109.5

109.5

109.5

109.5

109.5

109.5

109.5

109.5

110.66 (12)

110.67 (12)

$108.29(12)$

109.1 


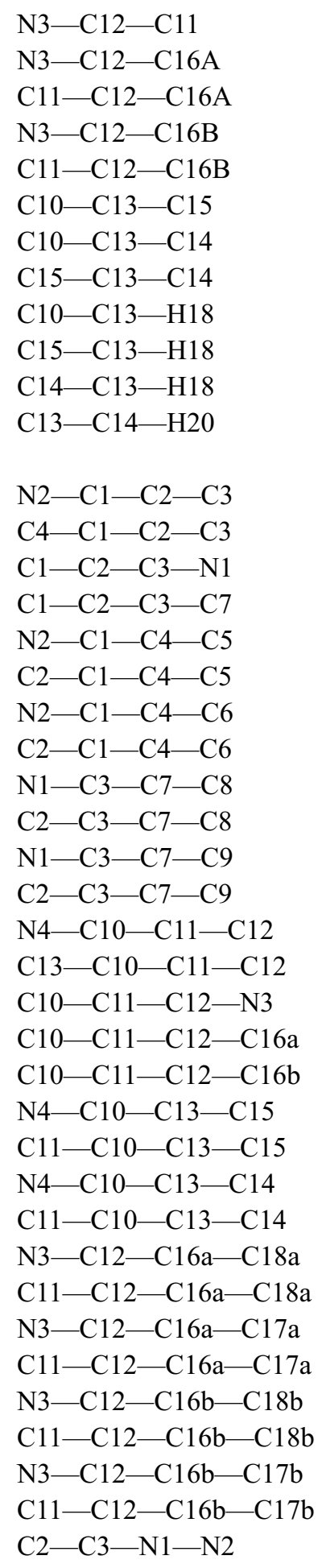

\section{$107.36(13)$}

$123.61(13)$

$129.03(15)$

$123.61(13)$

$129.03(15)$

$111.21(14)$

$111.11(15)$

$111.14(17)$

107.7

107.7

107.7

109.5

$-0.15(18)$

$-178.52(15)$

$0.01(18)$

$-178.32(16)$

$-103.02(17)$

$75.1(2)$

$134.38(16)$

$-47.5(2)$

$132.58(18)$

$-49.3(2)$

$-104.46(18)$

$73.6(2)$

0.08 (19)

$179.94(16)$

$0.00(18)$

$-179.54(16)$

$-179.54(16)$

$-120.42(18)$

59.7 (2)

$115.21(18)$

$-64.6(2)$

$135.3(3)$

$-45.3(4)$

$-101.6(3)$

$77.9(3)$

$-139.2(6)$

$40.3(6)$

100.3 (4)

$-80.2(5)$

0.14 (17)

$$
\begin{aligned}
& \mathrm{N} 1-\mathrm{B}-\mathrm{H} 1 \\
& \mathrm{~N} 2-\mathrm{B}-\mathrm{H} 1 \\
& \mathrm{C} 3-\mathrm{N} 1-\mathrm{N} 2 \\
& \mathrm{C} 3-\mathrm{N} 1-\mathrm{B} \\
& \mathrm{N} 2-\mathrm{N} 1-\mathrm{B} \\
& \mathrm{C} 1-\mathrm{N} 2-\mathrm{N} 1 \\
& \mathrm{C} 1-\mathrm{N} 2-\mathrm{B}^{\mathrm{i}} \\
& \mathrm{N} 1-\mathrm{N} 2-\mathrm{B}^{\mathrm{i}} \\
& \mathrm{C} 12-\mathrm{N} 3-\mathrm{N} 4 \\
& \mathrm{C} 12-\mathrm{N} 3-\mathrm{B} \\
& \mathrm{N} 4-\mathrm{N} 3-\mathrm{B} \\
& \mathrm{C} 10-\mathrm{N} 4-\mathrm{N} 3
\end{aligned}
$$

$\mathrm{C} 7-\mathrm{C} 3-\mathrm{N} 1-\mathrm{N} 2$

$\mathrm{C} 2-\mathrm{C} 3-\mathrm{N} 1-\mathrm{B}$

$\mathrm{C} 7-\mathrm{C} 3-\mathrm{N} 1-\mathrm{B}$

$\mathrm{N} 3-\mathrm{B}-\mathrm{N} 1-\mathrm{C} 3$

$\mathrm{N} 2 \mathrm{i}-\mathrm{B}-\mathrm{N} 1-\mathrm{C} 3$

$\mathrm{N} 3-\mathrm{B}-\mathrm{N} 1-\mathrm{N} 2$

$\mathrm{N} 2{ }^{\mathrm{i}}-\mathrm{B}-\mathrm{N} 1-\mathrm{N} 2$

$\mathrm{C} 2-\mathrm{C} 1-\mathrm{N} 2-\mathrm{N} 1$

$\mathrm{C} 4-\mathrm{C} 1-\mathrm{N} 2-\mathrm{N} 1$

$\mathrm{C} 2-\mathrm{C} 1-\mathrm{N} 2-\mathrm{B}^{\mathrm{i}}$

$\mathrm{C} 4-\mathrm{C} 1-\mathrm{N} 2-\mathrm{B}^{\mathrm{i}}$

$\mathrm{C} 3-\mathrm{N} 1-\mathrm{N} 2-\mathrm{C} 1$

$\mathrm{B}-\mathrm{N} 1-\mathrm{N} 2-\mathrm{C} 1$

$\mathrm{C} 3-\mathrm{N} 1-\mathrm{N} 2-\mathrm{B}^{\mathrm{i}}$

$\mathrm{B}-\mathrm{N} 1-\mathrm{N} 2-\mathrm{B}^{\mathrm{i}}$

$\mathrm{C} 11-\mathrm{C} 12-\mathrm{N} 3-\mathrm{N} 4$

$\mathrm{C} 16 \mathrm{a}-\mathrm{C} 12-\mathrm{N} 3-\mathrm{N} 4$

$\mathrm{C} 16 \mathrm{~b}-\mathrm{C} 12-\mathrm{N} 3-\mathrm{N} 4$

$\mathrm{C} 11-\mathrm{C} 12-\mathrm{N} 3-\mathrm{B}$

$\mathrm{C} 16 \mathrm{a}-\mathrm{C} 12-\mathrm{N} 3-\mathrm{B}$

$\mathrm{C} 16 \mathrm{~b}-\mathrm{C} 12-\mathrm{N} 3-\mathrm{B}$

$\mathrm{N} 1-\mathrm{B}-\mathrm{N} 3-\mathrm{C} 12$

$\mathrm{N} 2 \mathrm{i}-\mathrm{B}-\mathrm{N} 3-\mathrm{C} 12$

$\mathrm{N} 1-\mathrm{B}-\mathrm{N} 3-\mathrm{N} 4$

$\mathrm{N} 2-\mathrm{B}-\mathrm{N} 3-\mathrm{N} 4$

$\mathrm{C} 11-\mathrm{C} 10-\mathrm{N} 4-\mathrm{N} 3$

$\mathrm{C} 13-\mathrm{C} 10-\mathrm{N} 4-\mathrm{N} 3$

C12-N3-N4- 10

$\mathrm{B}-\mathrm{N} 3-\mathrm{N} 4-\mathrm{C} 10$
109.1

109.1

$108.29(12)$

125.87 (12)

$125.66(11)$

$108.30(11)$

$126.05(12)$

125.43 (12)

$110.45(12)$

$130.79(12)$

$118.75(12)$

$105.56(12)$

$178.58(14)$

$-175.10(14)$

$3.3(2)$

61.64 (19)

$-176.89(13)$

$-112.79(14)$

8.7 (2)

$0.23(16)$

$178.73(13)$

$-174.58(14)$

3.9 (2)

$-0.23(16)$

$175.02(13)$

$174.62(13)$

$-10.1(2)$

-0.08 (17)

179.49 (14)

$179.49(14)$

$-178.88(14)$

0.7 (2)

0.7 (2)

60.88 (19)

$-59.2(2)$

$-117.84(14)$

$122.10(13)$

-0.13 (17)

$-179.99(14)$

0.13 (16)

179.09 (13)

Symmetry code: (i) $-x+1 / 2,-y+1 / 2,-z$. 